OPEN ACCESS

Edited by:

Hau-tieng Wu,

Duke University, United States

Reviewed by:

John Hughes,

University of Colorado Denver,

United States

Antonio Calcagni,

University of Trento, Italy

${ }^{*}$ Correspondence:

Mark A. Wolters

mwolters@fudan.edu.cn

Specialty section:

This article was submitted to Mathematics of Computation and

Data Science,

a section of the journal Frontiers in Applied Mathematics and

Statistics

Received: 22 March 2017 Accepted: 10 November 2017 Published: 27 November 2017

Citation:

Wolters MA (2017) Better Autologistic

Regression

Front. Appl. Math. Stat. 3:24. doi: 10.3389/fams.2017.00024

\section{Better Autologistic Regression}

\author{
Mark A. Wolters * \\ Shanghai Center for Mathematical Sciences, Fudan University, Shanghai, China
}

Autologistic regression is an important probability model for dichotomous random variables observed along with covariate information. It has been used in various fields for analyzing binary data possessing spatial or network structure. The model can be viewed as an extension of the autologistic model (also known as the Ising model, quadratic exponential binary distribution, or Boltzmann machine) to include covariates. It can also be viewed as an extension of logistic regression to handle responses that are not independent. Not all authors use exactly the same form of the autologistic regression model. Variations of the model differ in two respects. First, the variable coding - the two numbers used to represent the two possible states of the variables-might differ. Common coding choices are (zero, one) and (minus one, plus one). Second, the model might appear in either of two algebraic forms: a standard form, or a recently proposed centered form. Little attention has been paid to the effect of these differences, and the literature shows ambiguity about their importance. It is shown here that changes to either coding or centering in fact produce distinct, non-nested probability models. Theoretical results, numerical studies, and analysis of an ecological data set all show that the differences among the models can be large and practically significant. Understanding the nature of the differences and making appropriate modeling choices can lead to significantly improved autologistic regression analyses. The results strongly suggest that the standard model with plus/minus coding, which we call the symmetric autologistic model, is the most natural choice among the autologistic variants.

Keywords: probabilistic graphical models, Markov random fields, logistic regression, correlated binary random variables, spatial statistics

\section{INTRODUCTION}

The autologistic (AL) model is a probabilistic graphical model for multivariate binary data. It was introduced to the statistical literature by Besag $[1,2]$ and has also been developed by Kaiser and Cressie [3]. The same model appeared much earlier in statistical physics, where it is known as the Ising model (see e.g., $[4,5])$. It has been used extensively in image processing (e.g., [6-8]), and is also the model underlying the Boltzmann machine [9]. The same model has also been called the quadratic exponential binary distribution $[10,11]$, and, under that name, it has been described as the binary-variable analog of the multivariate normal distribution ([12]; see also [13]). As such, one may anticipate that the autologistic model will become increasingly useful as the number of complex, graph-structured data sets continues to grow.

When binary responses are observed along with covariate information, the autologistic model may be extended to become the autologistic regression (ALR) model. This model can be viewed as a natural extension of ordinary logistic regression to handle cases where responses are not independent. Under the ALR model, the responses follow an autologistic distribution, and the 
distribution's parameters are written in terms of a linear predictor involving the covariates. The ALR model has been used in a variety of fields, including ecology [14, 15], dentistry [16, 17], anthropology [18], materials science [19] and computer vision [20, 21].

As an example, consider the Hydrocotyle vulgaris data shown in Figure 1. These data were derived from the work of Carl and Kühn [22] and re-analyzed by Bardos et al. [23]. The responses are presence or absence of plant species $H$. vulgaris in a regular grid of 2,995 cells covering Germany. The covariate is altitude (in hundreds of meters), recorded as a number from 0 to 18.23. From the figure it is clear that altitude is inversely related to species presence, and also that the observed responses are spatially correlated. Simple logistic regression provides estimated probabilities that appear realistic, but samples drawn from the fitted logistic model have more noise than the observed response. Explicitly modeling spatial association through an ALR model can potentially improve goodness-of-fit and give a better evaluation of the true effect of altitude.

If an analyst wishes to perform autologistic regression on data such as these, they are faced with two choices about the structure of the model: coding and centering.

Coding refers to the pair of numbers used to represent the two possible states or levels of a binary variable. Here the two levels will be referred to as "low" and "high." Since binary outcomes are usually categorical, not numeric, the analyst may freely choose two values, $\ell$ and $h$, to represent the two states. Standard choices are the zero/one coding, $\{0,1\}$, used almost universally in the statistics literature, and the plus/minus coding, $\{-1,1\}$, used customarily in physics and also common in image processing.

Centering refers to the presence or absence of a particular term in the model formulae. Caragea and Kaiser [24] observed that the original ALR model provides regression coefficient estimates that are not easy to interpret. They proposed the centered model to correct this problem, and recommended that it become the default for future use. This viewpoint was furthered by Hughes et al. [25], who expanded on inferential and computational aspects of ALR, using the centered model exclusively.

One may, then, refer to two types of AL/ALR model: those with the centering adjustment (centered models), and those without it (standard models). Additionally, each type may be used with any chosen coding, leading to an infinite number of model variants (a term that will be used for a particular combination of type and coding).

The present work provides a detailed study of the different AL and ALR variants. It is confirmed that all variants are equivalent in the AL case, although the way they depend on their parameters varies widely. More interestingly, equivalence does not hold in the ALR case. The centered and standard ALR models are distinct, non-nested families of probability distributions. Even within ALR models of the same type, changing the coding will generally change its probabilistic structure. The differences among models can be large both qualitatively and quantitatively, certainly large enough to be of practical consequence.

The extant literature on autologistic regression does not contain a similar investigation of the impact of coding and centering. Indeed, it appears to demonstrate ambiguity about the importance of these two choices. Consider the following:

1. Some research communities use $\{0,1\}$ coding, and others use $\{-1,1\}$. At the same time, the author is not aware of a single article in which a justification was given for choosing one coding over the other in an ALR model.

2. In the literature, ALR models with different codings take the same algebraic form; a coding change is effected by simply plugging different numbers into the same formulae. This is done despite the well-known fact (repeated in Appendix A) that this operation is not, in general, equivalent to properly transforming the random variables.

3. The key references for the centered model [24, 25] both refer to it as an autologistic model with a "centered parameterization," and cite improved parameter interpretation as its main advantage.

4. The centering adjustment was motivated by analogy with Gaussian models, where both centered and uncentered models are part of the same distribution family.

Points 1 and 2 suggest that many researchers view the choice of coding in an ALR model as trivial or inconsequential. This misconception might arise because the coding change is a seemingly innocuous linear transformation of the responses, one which is trivial in the AL model. Points 3 and 4 show how a reader might come to assume that centered and standard ALR models
A

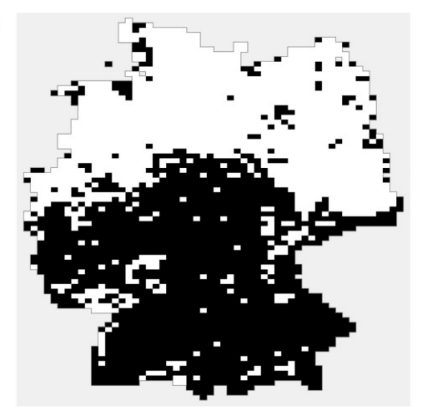

B

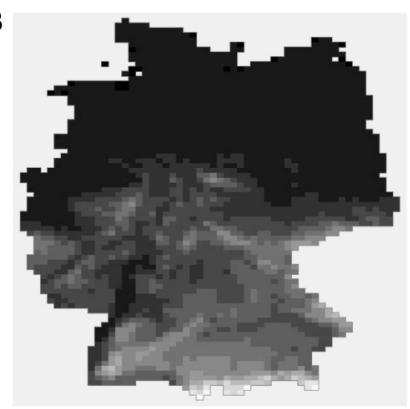

C

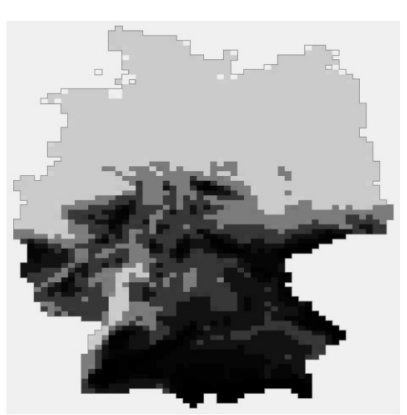

FIGURE 1 | The H. vulgaris data. (A) Observed presence/absence data (white indicates presence, which is taken to be the "high" level). (B) Value of the covariate, altitude. (C) Predicted probabilities from logistic regression. In (B,C), lighter shades of gray indicate larger values. 
are equivalent to one another, differing only by a parameter transformation.

The current work resolves the above misconceptions, and should help analysts develop better autologistic regression models by understanding the consequences of their modeling choices.

Throughout this research, one group of equivalent variants was repeatedly found to have unique and attractive properties. It is the set of standard models with coding that sums to zero, like $\{-h, h\}$. This model will be referred to as the symmetric autologistic model. It resolves the problems with the standard zero/one-coded model in a manner that is simpler and more natural than the centered model, and it is conceptually more appealing as a generative model for binary data.

The results are developed as follows. Section 2 provides more detail on the AL and ALR models, in both standard and centered forms. It also gives a general form of the model that includes both model types under arbitrary coding. The general form is used in section 3 to establish several theoretical results, including results about model equivalence and behavior in the limit as the inter-variable association increases. Section 4 provides numerical examples that demonstrate the differences among the model variants, while also illustrating and corroborating the theory. The $H$. vulgaris data is also analyzed at the end of section 4 . Section 5 summarizes the results and provides further discussion about the symmetric model.

\section{AUTOLOGISTIC MODELS}

This section lays out details of the AL and ALR models under different coding and centering choices. Because the notation and terminology used to describe these models varies considerably across disciplines, it is not assumed that every reader is familiar with the way the models are developed here. A somewhat expository tone is taken in this section.

\subsection{Markov Random Fields}

The AL model is best understood as a Markov random field (MRF) of binary random variables. MRFs provide a general framework for modeling collections of random variables, where the dependence among the variables is encoded by an undirected graph. We are interested in the binary case.

Let $\mathbf{Z}=\left[Z_{1}, Z_{2}, \ldots, Z_{n}\right]^{T}$ be a vector of $n$ dichotomous random variables (with coding as yet unspecified). Associated with $\mathbf{Z}$ is a graph $\mathcal{G}=(\mathcal{V}, \mathcal{E})$ having vertices $\mathcal{V}=\{1,2, \ldots, n\}$ (also called nodes; one for each variable) and edges $\mathcal{E}=$ $\{(i, j): i<j, i \sim j\}$, where $i, j \in\{1,2, \ldots, n\}$, and $i \sim j$ means variables $i$ and $j$ share an edge. Variables that are joined by an edge are called neighbors of one another.

The role of the graph is to define conditional independence statements. Specifically, let $\mathbf{Z}_{-i}$ represent all of the variables except for the $i$ th, and let $\mathbf{Z}_{j \sim i}$ represent all of the neighbors of $Z_{i}$ in the graph. In an MRF, each variable is conditionally independent of the others, given its neighbors: $\operatorname{Pr}\left(Z_{i}=z_{i} \mid \mathbf{Z}_{-i}\right)=\operatorname{Pr}\left(Z_{i}=z_{i} \mid \mathbf{Z}_{j \sim i}\right)$.
The joint distribution of an MRF can be expressed as a Boltzmann (or Gibbs) distribution:

$$
f(\mathbf{z})=\frac{1}{c} e^{Q(\mathbf{z})},
$$

where $Q(\mathbf{z})$ is called the negpotential function and the normalizing constant $c$ is known as the partition function. It is also common for $Q(\mathbf{z})$ to be referred to as an energy function, in which case it is usually preceded by a negative sign, and sometimes divided by a temperature parameter.

The negpotential function for any MRF can be expressed as a sum of functions of cliques of variables (that is, groups of fully connected vertices in the graph). Letting $\mathcal{M}$ represent the set of cliques, we have

$$
Q(\mathbf{z})=\sum_{m \in \mathcal{M}} \psi_{m}\left(\mathbf{z}_{m}\right)
$$

The sum consists of one function per clique, with the $m$ th function depending only on the variables that are part of the $m$ th clique. This fundamental result is known as the HammersleyClifford theorem; see Besag [2, 26] and Kaiser and Cressie [3] for more details and technical conditions on this MRF-Gibbs equivalence.

The high degree of generality of Equations (1) and (2) makes MRFs attractive for modeling complex associations. The graph structure determines which variables appear together in the $\psi_{m}$ functions, and the the functions themselves can be specified to control which arrangements of variables get greater probability mass. The price paid for this flexibility is computational: for most practical problems the partition function can not be computed in reasonable time.

We will subsequently focus on the particular form of the negpotential function used in the autologistic model. Our development of the AL model is consistent with that given in Hughes et al. [25], which also gives an excellent review of the computational aspects of inference with the AL model. For more on MRFs and graphical models in general, see e.g., [27-30].

\subsection{Standard and Centered Models with Zero/One Coding}

Both the standard and centered models have been developed under the assumption of $\{0,1\}$ coding. This section describes these models under the same assumption.

The autologistic model is a member of the class of so-called pairwise MRFs, where only cliques of size one or two have nonzero contribution to the negpotential function. The standard model has negpotential function

$$
Q_{\mathrm{std}}(\mathbf{z})=\sum_{i \in \mathcal{V}} \alpha_{i} z_{i}+\sum_{(i, j) \in \mathcal{E}} \lambda_{i j} z_{i} z_{j} .
$$

The right hand side of Equation (3) contains two sums. The first is over all vertices in the graph, and includes each individual variable's contributions to the probability mass function (PMF). The second is over all edges in the graph, and includes the contribution of each pair of neighbor variables. Values $\left\{\alpha_{i}\right\}$ and 
$\left\{\lambda_{i j}\right\}$ are called the unary and pairwise parameters, respectively. Note that if $\lambda_{i j}=0, \forall i, j$, the PMF factorizes and $Z_{1}, \ldots, Z_{n}$ are mutually independent.

The MRF-Gibbs equivalence makes it possible to specify the AL model through its conditional distributions. The conditional distributions are also used to write the conditional logit form of the model. Let $\pi_{i}^{*}=\operatorname{Pr}\left(Z_{i}=1 \mid \mathbf{Z}_{-i}\right)$. Then it can be shown that the standard model has conditional log odds

$$
\operatorname{logit}\left(\pi_{i}^{*}\right)=\log \left(\frac{\pi_{i}^{*}}{1-\pi_{i}^{*}}\right)=\alpha_{i}+\sum_{j \sim i} \lambda_{i j} z_{j}
$$

where $\sum_{j \sim i}$ denotes the sum over all $j$ that are neighbors of $i$. The log odds depends on the $i$ th variable's unary parameter plus a weighted sum of its neighbors' values. Increasing $\alpha_{i}$ or having more neighbors that take value 1 will increase the odds of observing a 1 in location $i$.

Inspection of Equation (4) hints at the parameter interpretation problem noted in Caragea and Kaiser [24]. The influence of the neighbor states on the conditional logit is asymmetric. Any neighbors taking value 0 contribute nothing to the conditional log odds, while neighbors with value 1 increase the log odds. No configuration of neighbors can cause $\operatorname{logit}\left(\pi_{i}^{*}\right)$ to decrease below the value of $\alpha_{i}$. This effectively couples the unary and pairwise parameters' effects.

The centered autologistic model was proposed as a modified form to ameliorate this problem. The centered model has conditional logit form

$$
\operatorname{logit}\left(\pi_{i}^{*}\right)=\alpha_{i}+\sum_{j \sim i} \lambda_{i j}\left(z_{j}-\mu_{j}^{*}\right)
$$

where the centering adjustment, $\mu_{j}^{*}$, is the expectation of the $j$ th variable under the assumption of independence:

$$
\mu_{j}^{*}=\mathrm{E}\left[Z_{j} \mid \lambda_{i j}=0, \forall i, j\right]=\frac{e^{\alpha_{j}}}{1+e^{\alpha_{j}}}
$$

\subsection{A General Form}

We now drop the requirement of $\{0,1\}$ coding, and express the autologistic model in a way that uses arbitrary coding and includes both centered and standard types as special cases. This may be done by defining the conditional distributions in a coding-agnostic manner, and then deriving the joint mass function using the MRF formalism. If the derivation is done for the centered case, any desired autologistic variant can be obtained afterwards by fixing the coding and either retaining the centering adjustment or setting it to zero. Details of the derivation are given in Appendix B, with only the results shown here.

Let the low and high values of the coding be $\ell$ and $h$, respectively (with $\ell<h$ ). First consider the centering adjustment for the $j$ th variable, which is denoted by $\mu_{j}$ in this general setting. For standard models, $\mu_{j}=0$. For centered models, $\mu_{j}$ is the mean of $Z_{j}$ under the assumption of independence:

$$
\begin{aligned}
\mu_{j} & =E\left[Z_{j} \mid \lambda_{i j}=0, \forall i, j\right] \\
& =\ell \operatorname{Pr}\left(Z_{j}=\ell \mid \forall \lambda_{i j}=0\right)+h \operatorname{Pr}\left(Z_{j}=h \mid \forall \lambda_{i j}=0\right) \\
& =\frac{\ell e^{\ell \alpha_{j}}+h e^{h \alpha_{j}}}{e^{\ell \alpha_{j}}+e^{h \alpha_{j}}} .
\end{aligned}
$$

The conditional forms of the model involve a term that is a sum of neighbor contributions. To simplify notation, define this neighbor sum for the $i$ th variable to be

$$
s_{i}=\sum_{j \sim i} \lambda_{i j}\left(z_{j}-\mu_{j}\right)
$$

Then the conditional PMF of $Z_{i}$, given all other $Z$ values, is

$$
\operatorname{Pr}\left(Z_{i}=z_{i} \mid \mathbf{Z}_{-i}\right)=\frac{\exp \left(z_{i}\left(\alpha_{i}+s_{i}\right)\right)}{\exp \left(\ell\left(\alpha_{i}+s_{i}\right)\right)+\exp \left(h\left(\alpha_{i}+s_{i}\right)\right)}
$$

and, letting $\pi_{i}=\operatorname{Pr}\left(Z_{i}=h \mid \mathbf{Z}_{-i}\right)$, the log odds form of the model is

$$
\operatorname{logit}\left(\pi_{i}\right)=(h-\ell)\left(\alpha_{i}+s_{i}\right) .
$$

Note that Equations (5) and (6) are special cases of Equations (10) and (7), with $\ell=0$ and $h=1$.

Finally, the joint PMF can be derived from the conditional form. To minimize ambiguity, it is included as a definition.

DEFINITION 1 (autologistic model). Let $\mathbf{Z}$ be a vector of $n$ dichotomous random variables with low and high values coded $\ell$ and $h$, respectively. Under the autologistic model, $\mathbf{Z}$ has PMF

$$
f_{\mathbf{Z}}(\mathbf{z} ; \boldsymbol{\alpha}, \boldsymbol{\Lambda}) \propto \exp \left(\mathbf{z}^{T} \boldsymbol{\alpha}-\mathbf{z}^{T} \boldsymbol{\Lambda} \boldsymbol{\mu}_{\boldsymbol{\alpha}}+\frac{1}{2} \mathbf{z}^{T} \boldsymbol{\Lambda} \mathbf{z}\right),
$$

where $\boldsymbol{\Lambda}$ is an $n \times n$ symmetric matrix with $(i, j)$ th and $(j, i)$ th elements equal to $\lambda_{i j}$ if $i \sim j$, and equal to zero otherwise; and

$\mu_{\alpha}=\left\{\begin{array}{cc}0 & \text { for a standard model } \\ {\left[\mu_{1}^{\alpha} \cdots \mu_{n}^{\alpha}\right]^{T} \text { with } \mu_{j}^{\alpha}=\frac{\ell e^{\ell \alpha_{j}}+h e^{h \alpha_{j}}}{e^{\ell \alpha_{j}}+e^{h \alpha_{j}}} \text { for a centered model }}\end{array}\right.$

Call coefficient $\boldsymbol{\alpha}=\left[\alpha_{1}, \ldots, \alpha_{n}\right]^{T}$ the unary parameter, and call $\boldsymbol{\Lambda}$ the association matrix. As a compact notation, refer to the standard model as $\mathrm{S}_{\ell, h}(\boldsymbol{\alpha}, \boldsymbol{\Lambda})$ and to the centered model as $\mathrm{C}_{\ell, h}(\boldsymbol{\alpha}, \boldsymbol{\Lambda})$.

This definition gives the negpotential function in matrix-vector form. The association matrix $\boldsymbol{\Lambda}$ has the same pattern of nonzero elements as the adjacency matrix, $\mathbf{A}$, of the graph. It is common in applications to assume that the association parameter takes a constant value $\lambda$ throughout the graph, in which case $\boldsymbol{\Lambda}=\lambda \mathbf{A}$. This will be called the simple smoothing assumption.

Equation (11) looks the same as the (zero/one coded, centered) PMF in Hughes et al. [25], but note that in the present case the centering term is a function of not only the unary parameter, but of $\ell$ and $h$ as well. The vector of centering 
adjustments, $\boldsymbol{\mu}_{\boldsymbol{\alpha}}$, has been written with a subscript as a reminder of its dependence on $\boldsymbol{\alpha}$.

Equations (9), (10), and (11) express the model in different ways. To reiterate, the standard model is obtained by setting $\boldsymbol{\mu}=\mathbf{0}$, and any desired coding can be obtained by setting $\{\ell, h\}$ accordingly.

\subsection{Autologistic Regression}

The AL model just presented does not include covariate effects. The usual way to introduce covariates is to replace the unary parameters by linear predictors. This is formalized in a definition.

DEFINITION 2 (autologistic regression model). Let $\mathbf{Z}$ and $f_{\mathbf{Z}}$ be the same as in Definition 1. Let $\mathbf{X}$ be an $n \times p$ matrix of covariate information (including intercept if desired), with $p<n$. Define $\mathbf{x}_{i}^{T}$ to be the ith row of $\mathbf{X}$, and let $\boldsymbol{\beta}$ be a p-vector of coefficients. Then the autologistic regression model for $\mathbf{Z}$ is $f_{\mathbf{Z}}(\mathbf{z} ; \mathbf{X} \boldsymbol{\beta}, \boldsymbol{\Lambda})$. In other words, it is model (11) with $\alpha_{i} \equiv \mathbf{x}_{i}^{T} \boldsymbol{\beta}$.

The ALR model replaces the $n$ unary parameters $\left\{\alpha_{i}\right\}$ by the $p$ regression coefficients $\beta$. The covariates appear only in the unary parameter, as part of the linear relationship $\boldsymbol{\alpha}=\mathbf{X} \boldsymbol{\beta}$. Once the linear predictor values are fixed, the ALR model becomes an AL model. Because of this, the qualitative behavior of an ALR model is the same as that of an AL model, and if we understand how to interpret $\boldsymbol{\alpha}$, the interpretation of $\boldsymbol{\beta}$ will be the same. For this reason the results and analyses of sections 3 and 4 are based primarily on the AL model, unless they specifically relate to the regression parameters.

\subsection{About the Parameters}

Regardless of centering or coding choice, the graphical nature of the AL/ALR model and its mathematical form both strongly suggest a certain parameter interpretation. The unary parameters control each variable's inherent tendency to take one value or the other. All else being equal, large positive values of $\alpha_{i}$ (or $\mathbf{x}_{i}^{T} \boldsymbol{\beta}$ ) should indicate a high chance to observe the $h$ state at location $i$, and large negative values should indicate a high chance of $\ell$. The pairwise parameters control the amount of influence neighbor variables have on each other, and therefore control the association structure. Following Towner et al. [18], call an edge concordant if the two vertices it joins both take the same state, and discordant if they differ. Large positive $\lambda_{i j}$ values should increase the chance that the edge joining $Z_{i}$ and $Z_{j}$ is concordant. Negative $\lambda_{i j}$ values promote discordant edges. It is assumed throughout this work that all $\lambda_{i j} \geq 0$, since positive association is of greater practical interest.

All variants of the model share the property that $\lambda_{i j}=$ $0, \forall i, j$ corresponds to statistical independence. The model's behavior under independence will be called its endogenous structure, and the probability that variable $i$ takes its high value, under independence, will be called its endogenous probability, $p_{i}$. Other authors $[24,25]$, in the context of spatial data with smoothly-varying covariates, have used the term "large scale" structure to refer to the model's predictions under independence. "Endogenous" has been preferred here because the graph need not be spatially referenced, nor do the unary parameter values need to be locally smooth.
The explicit goal of the centered ALR model [24, p. 286] was to make the marginal probabilities remain close to the endogenous probabilities even when $\lambda$ is nonzero, so that the regression coefficients can be said to control the marginal probabilities regardless of association level. Marginal structure is only part of the story, however: the model describes a distribution over the $2^{n}$ possible outcomes in its sample space. Each outcome corresponds to a configuration, which is the arrangement of high and low states, irrespective of their numerical coding.

DEFINITION 3 (configuration). The term configuration may be used to refer to either a) any particular outcome in the sample space of a dichotomous random vector, or b) the locations of an outcome's high-and low-valued elements. Two binary vectors with different coding represent the same configuration if the locations of their high-and low-valued elements coincide.

If we repeatedly sample from an $\mathrm{AL}$ model with a nonzero association matrix, the configurations that we observe will be the result of a trade-off between the endogenous part of the model (which depends on $\boldsymbol{\alpha}$ alone) and the association effects (depending on $\boldsymbol{\Lambda}$ ) propagating through the graph. It is crucial, then, to understand how the distribution of configurations changes to reflect this trade-off as the association is increased from zero. This understanding is necessary for proper parameter interpretation, and also for assessing the model as a reasonable data-generating process to approximate real-world phenomena.

\section{THEORETICAL RESULTS}

This section provides theorems that help to discern the differences among the AL/ALR model variants. The first results consider whether or not different variants are equivalent to each other. Subsequent theorems address the behavior of the different variants in the limit as the association parameter grows large. The section closes with a result on convexity of the pseudolikelihood function.

\subsection{Equivalence of AL models}

There is potential for confusion when comparing two models that may differ in both their variable coding and their parametrization. To minimize this, model equivalence is first defined in a coding-independent way.

DEFINITION 4 (equivalent models). Let $f_{1}\left(\mathbf{z} ; \boldsymbol{\theta}_{1}\right)$ and $f_{2}\left(\mathbf{y} ; \boldsymbol{\theta}_{2}\right)$ be two joint PMFs for $n$ binary outcomes, where $\mathbf{z}$ and $\mathbf{y}$ need not have the same coding. Model $f_{1}$ is equivalent to model $f_{2}$ if, for any $\boldsymbol{\theta}_{2}$, there exists a $\boldsymbol{\theta}_{1}^{*}$ such that $f_{1}\left(\mathbf{z} ; \boldsymbol{\theta}_{1}^{*}\right)=f_{2}\left(\mathbf{y} ; \boldsymbol{\theta}_{2}\right)$ whenever $\mathbf{z}$ and $\mathrm{y}$ represent the same configuration.

Equivalence means that given $f_{2}$ with fixed $\boldsymbol{\theta}_{2}$, there is always a parameter setting of $f_{1}$ that makes the two models assign the same probability distribution over the $2^{n}$ configurations. The following theorem shows that all variants of the autologistic model are equivalent to the standard model. It only applies to AL models, not to ALR models.

THEOREM 1 (equivalence of AL models). Let $f(\cdot ; \boldsymbol{\phi}, \boldsymbol{\Omega})$ be an autologistic model (either centered or standard), with variable 
coding $\{L, H\}$. Then $f$ is equivalent to $\mathrm{S}_{\ell, h}(\boldsymbol{\alpha}, \boldsymbol{\Lambda})$, a standard model with coding $\{\ell, h\}$. Furthermore, the parameter transformation that makes it equivalent is

$$
\begin{aligned}
\boldsymbol{\alpha} & =a\left(\boldsymbol{\phi}-\boldsymbol{\Omega} \boldsymbol{\mu}_{\boldsymbol{\phi}}+b \boldsymbol{\Omega} \mathbf{1}\right) \\
\boldsymbol{\Lambda} & =a^{2} \boldsymbol{\Omega},
\end{aligned}
$$

where $a=\frac{H-L}{h-\ell}, b=L-a \ell$, and $\mathbf{1}$ is a vector of ones.

PROOF: Let $\mathbf{Y}$ be a vector of binary random variables coded $\{L, H\}$, and let $f_{\mathbf{Y}}(\mathbf{y} ; \boldsymbol{\phi}, \boldsymbol{\Omega})$ be an autologistic model of either type. Let $\mathbf{Z}$ be a vector of binary random variables of the same dimension as $\mathbf{Y}$, coded $\{\ell, h\}$ and having standard autologistic PMF $f_{\mathbf{Z}}=\mathrm{S}_{\ell, h}(\boldsymbol{\alpha}, \boldsymbol{\Lambda})$. Equivalence requires that $f_{\mathbf{Y}}(\mathbf{y})=f_{\mathbf{Z}}(\mathbf{z})$ whenever $\mathbf{y}$ and $\mathbf{z}$ represent the same configuration. But in that case, there is a one-to-one transformation linking the two vectors: $\mathbf{y}=a \mathbf{z}+b \mathbf{1}$, with $a$ and $b$ as in the theorem. We have equivalence, then, if we can choose $\boldsymbol{\alpha}$ and $\boldsymbol{\Lambda}$ such that $f_{\mathbf{Z}}(\mathbf{z} ; \boldsymbol{\alpha}, \boldsymbol{\Lambda})=f_{\mathbf{Y}}(a \mathbf{z}+$ $b \mathbf{1} ; \boldsymbol{\phi}, \boldsymbol{\Omega})$ for all $\mathbf{z}$.

From Equation (11), the negpotential function for the standard model $f_{\mathbf{Z}}(\mathbf{z})$ is

$$
Q_{\mathbf{Z}}(\mathbf{z} ; \boldsymbol{\alpha}, \boldsymbol{\Lambda})=\mathbf{z}^{T} \boldsymbol{\alpha}+\frac{1}{2} \mathbf{z}^{T} \boldsymbol{\Lambda} \mathbf{z} .
$$

For $f_{\mathbf{Y}}(a \mathbf{z}+b \mathbf{1})$, which could be of either centered or standard type, the negpotential function is

$$
\begin{aligned}
Q_{\mathbf{Y}}(a \mathbf{z}+b \mathbf{1} ; \boldsymbol{\phi}, \boldsymbol{\Omega})= & (a \mathbf{z}+b \mathbf{1})^{T}\left(\boldsymbol{\phi}-\boldsymbol{\Omega} \boldsymbol{\mu}_{\boldsymbol{\phi}}\right) \\
& +\frac{1}{2}(a \mathbf{z}+b \mathbf{1})^{T} \boldsymbol{\Omega}(a \mathbf{z}+b \mathbf{1}) \\
= & a \mathbf{z}^{T}\left(\boldsymbol{\phi}-\boldsymbol{\Omega} \boldsymbol{\mu}_{\boldsymbol{\phi}}+b \boldsymbol{\Omega} \mathbf{1}\right) \\
& +\frac{1}{2} a^{2} \mathbf{z}^{T} \boldsymbol{\Omega} \mathbf{z}+w,
\end{aligned}
$$

where the last line was obtained by multiplying out the products and moving terms free of $\mathbf{z}$ into the constant $w$.

The two PMFs are specified only up to a proportionality constant, so equivalence of the PMFs holds if and only if $\exp \left(Q_{\mathbf{Z}}(\mathbf{z} ; \boldsymbol{\alpha}, \boldsymbol{\Lambda})\right)$ and $\exp \left(Q_{\mathbf{Y}}(a \mathbf{z}+b \mathbf{1} ; \boldsymbol{\phi}, \boldsymbol{\Omega})\right.$ can be made proportional to each other-or alternatively, if the two negpotential functions can be made to differ by at most a $\mathbf{z}$-free additive constant. For the difference of the right-hand sides of Equations (15) and (16) to be $\mathbf{z}$-free, we must have

$$
\mathbf{z}^{T}\left[\boldsymbol{\alpha}-a\left(\boldsymbol{\phi}-\boldsymbol{\Omega} \boldsymbol{\mu}_{\boldsymbol{\phi}}+b \boldsymbol{\Omega} \mathbf{1}\right)\right]+\frac{1}{2} \mathbf{z}^{T}\left(\boldsymbol{\Lambda}-a^{2} \boldsymbol{\Omega}\right) \mathbf{z}=0 .
$$

Since this must hold for all $\mathbf{z}$, the coefficients of both the linear and quadratic terms must be zero, which leads us to the transformation given in the theorem. The existence of the transformation proves that the models are equivalent, and because the result does not depend on the particular form of $\boldsymbol{\mu}_{\boldsymbol{\phi}}$, it holds regardless of whether $f_{\mathbf{Y}}$ is a centered or standard model.

If $f$ in Theorem 1 is a standard autologistic model, $\mu_{\phi}=0$ and Equation (13) may be solved explicitly for either $\boldsymbol{\alpha}$ or $\boldsymbol{\phi}$.
This shows that there is a one-to-one correspondence between standard models with different codings. When $f$ is a centered model, however, Equation (13) is a system of nonlinear equations in $\phi$, and since the inverse transformation can not be analytically determined, it remains unclear if the mapping between $\alpha$ and $\phi$ is one-to-one. The implicit function theorem of vector calculus [31, section 12.8] could be used to show that the inverse exists for all $\boldsymbol{\alpha}$, but it is not straightforward to show that the Jacobian determinant of transformation (13) is always nonzero. Consequently, Theorem 1 only shows that every autologistic model is equivalent to any chosen model of standard type; it falls short of claiming full one-to-one correspondence between all AL model variants. Nevertheless, it may be conjectured that such a correspondence does exist, and system (13) has been successfully solved for $\boldsymbol{\phi}$ numerically, using a fixed-point iteration scheme.

\subsection{Non-equivalence of ALR Models}

The next theorem implies that the equivalence observed among the autologistic models does not, in general, carry over to autologistic regression models.

THEOREM 2 (condition for equivalence of ALR models). Let $\mathbf{X}$ be an $n \times p$ matrix with $n>p$, and let $f_{1}(\cdot ; \mathbf{X} \boldsymbol{\gamma}, \boldsymbol{\Omega})$ and $f_{2}(\cdot ; \mathbf{X} \boldsymbol{\beta}, \boldsymbol{\Lambda})$ be two autologistic regression models, using coding $\{L, H\}$ and $\{\ell, h\}$, respectively. Then model $f_{2}$ is equivalent to $f_{1}$ if and only if $\boldsymbol{\beta}$ satisfies

$$
\mathbf{X} \boldsymbol{\beta}-a^{2} \boldsymbol{\Omega} \mu_{\mathbf{X} \boldsymbol{\beta}}=a \mathbf{X} \boldsymbol{\gamma}+a \boldsymbol{\Omega}\left(b \mathbf{1}-\boldsymbol{\mu}_{\mathbf{X} \boldsymbol{\gamma}}\right),
$$

where $a$ and $b$ are as defined in Theorem 1. This is an overdetermined system of equations in $\boldsymbol{\beta}$ (linear equations if $f_{2}$ is a standard model, and nonlinear if it is centered).

PROOF: The proof of Theorem 1 considered the equivalence of $f_{\mathrm{Y}}$, a centered AL model, and $f_{\mathrm{Z}}$, a standard AL model. Following exactly the same reasoning as that proof, but allowing $f_{\mathbf{Z}}$ to be a centered model, we find the transformation that makes $f_{\mathrm{Z}}$ equivalent to $f_{\mathrm{Y}}$ is

$$
\begin{aligned}
\boldsymbol{\alpha}-a^{2} \boldsymbol{\Omega} \boldsymbol{\mu}_{\boldsymbol{\alpha}} & =a\left(\boldsymbol{\phi}-\boldsymbol{\Omega} \boldsymbol{\mu}_{\boldsymbol{\phi}}+b \boldsymbol{\Omega} \mathbf{1}\right) \\
\boldsymbol{\Lambda} & =a^{2} \boldsymbol{\Omega}
\end{aligned}
$$

where $a$ and $b$ are as defined in Theorem 1 and $\boldsymbol{\mu}_{\boldsymbol{\alpha}}, \boldsymbol{\mu}_{\boldsymbol{\phi}}$ are as defined in Equation (12) (note that $\mu_{\alpha}$ uses $\{\ell, h\}$ coding, while $\boldsymbol{\mu}_{\boldsymbol{\phi}}$ uses $\left.\{L, H\}\right)$. The association matrices are straightforwardly transformed via Equation (19), but equivalence also requires that system (18) is consistent.

The ALR case discussed in the theorem is exactly the same, but with $\boldsymbol{\phi} \equiv \mathbf{X} \boldsymbol{\gamma}$ and $\boldsymbol{\alpha} \equiv \mathbf{X} \boldsymbol{\beta}$. Writing Equation (18) in terms of these regression parameters gives system (17). Model equivalence is the same as consistency of that system. As before, these results to not depend on the form of $\mu_{\alpha}$ or $\mu_{\phi}$, so they hold irrespective of whether either model is centered or standard.

The covariate matrix $\mathbf{X}$ in the theorem is assumed full rank, but is otherwise arbitrary. With no special structure for $\mathbf{X}$, system (17) will generally be inconsistent, meaning that model $f_{2}$ cannot 
be made equivalent to $f_{1}$. We have not proven that the system can never have a solution. Nonetheless, any practical concept of model equivalence should have equivalence hold regardless of the particular covariates observed, so in the following we declare that two models are not equivalent unless we can explicitly prove that the system (17) has a solution in $\beta$ for arbitrary $\mathbf{X}$.

Inspection of the system does reveal two situations where enough terms become zero to permit a solution for $\boldsymbol{\beta}$. The first is when the variables are independent, so that $\boldsymbol{\Omega}$ is a zero matrix and we fall back to ordinary logistic regression. In the notation of the theorem, the parameter transformation is $\beta=a \gamma$ in this case. Independence is a trivial case and henceforth it is assumed that $\boldsymbol{\Omega}$ is not zero. The second case is summarized as a corollary.

COROLLARY 2.1. If $f_{1}$ and $f_{2}$ in Theorem 2 are both standard models and their codings satisfy $\mathrm{Hl}=\mathrm{Lh}$, then the models are equivalent and the parameter transformation

$$
\boldsymbol{\beta}=a \boldsymbol{\gamma}, \quad \boldsymbol{\Lambda}=a^{2} \boldsymbol{\Omega}
$$

makes $f_{2}$ equivalent to $f_{1}$.

PROOF: Since both models are of standard type, $\mu_{\mathbf{X} \beta}=$ $\boldsymbol{\mu}_{\mathbf{X} \boldsymbol{\gamma}}=\mathbf{0}$. The restriction $H \ell=L h$ ensures that $b=0$. Then the system (17) has solution $\boldsymbol{\beta}=a \boldsymbol{\gamma}$, and the pairwise parameter transformation is $\boldsymbol{\Lambda}=a^{2} \boldsymbol{\Omega}$, as in Equation (19).

Corollary 2.1 refers to the case where two standard ALR models have codings that differ only by a positive scaling factor. In this case the models are equivalent. For example, standard ALR models with coding $\{0,1\}$ and $\{0,2\}$ are equivalent, as are models with coding $\{-2,4\}$ and $\{-4,8\}$. The $\{0,1\}$ and $\{-1,1\}$ codings, which are of most interest, do not satisfy this requirement. Thus ALR models with zero/one and plus/minus codings are not, in general, equivalent, even if both models are of standard type.

\subsection{Behavior with Strong Association}

Here we consider the behavior of the autologistic PMF under the simple smoothing assumption when $\lambda \rightarrow \infty$. As mentioned in section 2.5 , understanding the effect of increasing $\lambda$ is particularly helpful for understanding the model and its parameters. The question is first considered for a simple two-variable case, and then for the general case.

\subsubsection{The Two-Variable Case}

The $n=2$ case has the simplest nontrivial graph and is useful to study the limiting behavior in a simple situation. Figure 2 shows the graph. The variables are $Z_{1}$ and $Z_{2}$, with corresponding unary parameters $\alpha_{1}, \alpha_{2}$ and pairwise parameter $\lambda$. The coding is $\{\ell, h\}$. The figure also shows the joint PMF of the two variables as four probabilities in a $2 \times 2$ table. The following theorem gives the values of the probabilities in the limit of large association parameter, for all variants of the AL model.

THEOREM 3 (limiting probabilities, two-variable case). Let $Z_{1}$ and $Z_{2}$ be jointly distributed according to autologistic model $f_{\mathbf{Z}}$,

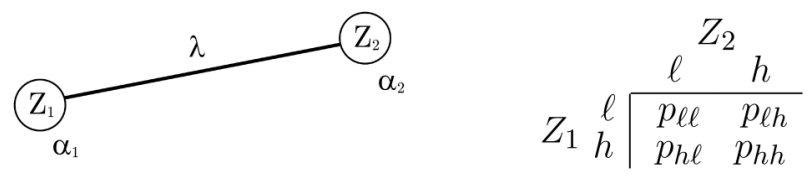

FIGURE 2 | The $n=2$ case with variables $Z_{1}$ and $Z_{2}$. (Left) The graph, showing the model parameters. (Right) The joint PMF.

with graph and probability table as shown in Figure 2. Let $p_{\ell \ell}^{*}, p_{\ell h}^{*}$, $p_{h \ell}^{*}$, and $p_{h h}^{*}$ be the limiting probabilities in the table as $\lambda \rightarrow \infty$.

a) If $f_{\mathrm{Z}}$ is a standard model and $\alpha_{1}, \alpha_{2}$ take any fixed values, then:

$$
\begin{aligned}
& \text { if } \ell+h>0, \quad p_{h h}^{*}=1 \\
& \text { if } \ell+h<0, \quad p_{\ell \ell}^{*}=1 \\
& \text { if } \ell+h=0, \quad p_{h h}^{*}=\frac{e^{h\left(\alpha_{1}+\alpha_{2}\right)}}{e^{\ell\left(\alpha_{1}+\alpha_{2}\right)}+e^{h\left(\alpha_{1}+\alpha_{2}\right)}} \text { and } p_{\ell \ell}^{*}=1-p_{h h}^{*}
\end{aligned}
$$

b) If $f_{\mathrm{Z}}$ is a centered model and $\ell, h$ take any fixed values, then:

$$
\begin{aligned}
& \text { if } \alpha_{1}+\alpha_{2}>0, p_{\ell \ell}^{*}=1 \\
& \text { if } \alpha_{1}+\alpha_{2}<0, \quad p_{h h}^{*}=1 \\
& \text { if } \alpha_{1}+\alpha_{2}=0, p_{h h}^{*}=p_{\ell \ell}^{*}=\frac{1}{2}
\end{aligned}
$$

PROOF: Considering Equation (11) with $n=2$, we can define the un-normalized PMF as

$$
g\left(z_{1}, z_{2}\right)=e^{\alpha_{1} z_{1}} e^{\alpha_{2} z_{2}} e^{\left(z_{1} z_{2}-\mu_{2} z_{1}-\mu_{1} z_{2}\right) \lambda}
$$

where $\mu_{1}$ and $\mu_{2}$ are the centering adjustments of $Z_{1}$ and $Z_{2}$ according to Equation (12). Then the probability of a particular configuration $\left(z_{1}, z_{2}\right)$ is

$$
\begin{aligned}
\operatorname{Pr} & \left.Z_{1}=z_{1}, Z_{2}=z_{2}\right) \\
& =\frac{g\left(z_{1}, z_{2}\right)}{g(\ell, \ell)+g(\ell, h)+g(h, \ell)+g(h, h)} \\
& =e^{\alpha_{1} z_{1}} e^{\alpha_{2} z_{2}}\left[\frac{g(\ell, \ell)+g(\ell, h)+g(h, \ell)+g(h, h)}{e^{\left(z_{1} z_{2}-\mu_{2} z_{1}-\mu_{1} z_{2}\right) \lambda}}\right]^{-1} .
\end{aligned}
$$

We are interested in the limiting value of this probability in eight cases: four configurations of $\left(z_{1}, z_{2}\right)$, for each of the two model types. Clearly, any of these probabilities will be nonzero only if the ratio in the square brackets above (call it $R$ ) remains finite as $\lambda \rightarrow \infty$. All of the results of the theorem are found by considering how this ratio behaves (as a function of $\alpha_{1}, \alpha_{2}, \ell$, and h) over the eight cases.

Consider for example the case of the $(h, h)$ configuration under the centered model. For this case, after some algebraic manipulations, we have

$$
\begin{aligned}
R= & e^{\ell\left(\alpha_{1}+\alpha_{2}\right)} e^{(\ell-h)\left(h+\ell-\mu_{1}-\mu_{2}\right) \lambda} \\
& +e^{\left(\ell \alpha_{1}+h \alpha_{2}\right)} e^{(\ell-h)\left(h-\mu_{2}\right) \lambda} \\
& +e^{\left(h \alpha_{1}+\ell \alpha_{2}\right)} e^{(\ell-h)\left(h-\mu_{1}\right) \lambda}+e^{h\left(\alpha_{1}+\alpha_{2}\right)} .
\end{aligned}
$$

The right hand side is a sum of four terms. The last term is finite and not a function of $\lambda$. The second and third terms approach zero as $\lambda \rightarrow \infty$, since $\ell<h$ and $h$ is greater than both $\mu_{1}$ and $\mu_{2}$. So the value of $p_{h h}^{*}$ depends on the sign of $h+\ell-\mu_{1}-\mu_{2}$. 
Using Equation (12) to write $\mu_{1}, \mu_{2}$ in terms of $h, \ell, \alpha_{1}$, and $\alpha_{2}$, it can be shown that

$$
\operatorname{sgn}\left(h+\ell-\mu_{1}-\mu_{2}\right)=\operatorname{sgn}\left(e^{\ell\left(\alpha_{1}+\alpha_{2}\right)}-e^{h\left(\alpha_{1}+\alpha_{2}\right)}\right),
$$

which equals $(1,-1,0)$ when $\alpha_{1}+\alpha_{2}$ is (less than, greater than, equal to) zero. From this we conclude the results about $p_{h h}^{*}$ in part b) of the theorem. The remaining results are obtained similarly by working out $R$ for the other cases and considering its limiting behavior.

The theorem shows that for the standard model, the limiting probabilities are only reasonable when the coding is symmetric around zero. If the sum of the coding values is nonzero, either $p_{\ell \ell}^{*}$ or $p_{h h}^{*}$ gets all of the probability mass, and which configuration gets the mass depends only on the coding, not on the unary parameters. When $\ell+h=0$, the limiting probabilities make sense: either configuration can occur, with weight that depends on $\alpha_{1}+\alpha_{2}$.

For the centered model, the limiting behavior does depend on $\alpha_{1}+\alpha_{2}$, but it does so in a counterintuitive way. For $\alpha_{1}+\alpha_{2} \neq$ 0 , the configuration that is opposite of the unary parameters' endogenous tendency receives all of the probability mass. Large positive values of the unary parameters (which should promote the occurrence of $h$ states) lead to $p_{\ell \ell}^{*}=1$ in the limit. Large negative values lead to $p_{h h}^{*}=1$. Only at the midpoint between these scenarios, $\alpha_{1}+\alpha_{2}=0$, does the limiting PMF take a reasonable form.

\subsubsection{The General Case}

In the general- $n$ case it relatively straightforward to determine the limiting probabilities for the symmetric model. Combined with the $n=2$ results it is possible to make the following statement.

THEOREM 4 (limiting probabilities, general case). Let $n$-vector $\mathrm{Z}$ be distributed according to standard autologistic model $\mathrm{S}_{\ell, h}(\boldsymbol{\alpha}, \lambda \mathbf{A})$ with $\ell+h=0$ and with a connected graph. Define $\ell=\ell \mathbf{l}$ and $\mathbf{h}=h \mathbf{1}$. Then the two configurations $\mathbf{z}=\ell$ and $\mathbf{z}=\mathbf{h}$ are the only ones with nonzero probability as $\lambda \rightarrow \infty$, and this holds regardless of the parameter values or the particulars of the graph. The limiting probabilities are

$$
p_{\mathbf{h}}^{*}=\frac{\exp \left(h \sum_{i=1}^{n} \alpha_{i}\right)}{\exp \left(h \sum_{i=1}^{n} \alpha_{i}\right)+\exp \left(\ell \sum_{i=1}^{n} \alpha_{i}\right)} \text { and } p_{\ell}^{*}=1-p_{\mathbf{h}}^{*}
$$

Furthermore, the standard model with $\ell+h=0$ is the only autologistic model variant for which more than one configuration has positive limiting probability regardless of $n$, the graph structure, or the parameter values.

PROOF: The standard model has PMF

$$
f(\mathbf{z})=\frac{\exp \left(\mathbf{z}^{T} \boldsymbol{\alpha}+\frac{\lambda}{2} \mathbf{z}^{T} \mathbf{A z}\right)}{\sum_{\mathbf{x} \in \mathcal{C}} \exp \left(\mathbf{x}^{T} \boldsymbol{\alpha}+\frac{\lambda}{2} \mathbf{x}^{T} \mathbf{A x}\right)}
$$

$$
\begin{aligned}
& =\frac{\exp \left(\mathbf{z}^{T} \boldsymbol{\alpha}\right)}{\sum_{\mathbf{x} \in \mathcal{C}} \exp \left(\mathbf{x}^{T} \boldsymbol{\alpha}\right) \exp \left(\frac{\lambda}{2} \mathbf{x}^{T} \mathbf{A x}\right) \exp \left(-\frac{\lambda}{2} \mathbf{z}^{T} \mathbf{A z}\right)} \\
& =\frac{\exp \left(\mathbf{z}^{T} \boldsymbol{\alpha}\right)}{\sum_{\mathbf{x} \in \mathcal{C}} \exp \left(\mathbf{x}^{T} \boldsymbol{\alpha}\right) \exp \left(\lambda \sum_{\mathcal{E}}\left(x_{i} x_{j}-z_{i} z_{j}\right)\right)},
\end{aligned}
$$

where $\mathcal{C}$ is the set of $2^{n}$ possible configurations. Letting $d_{i j}=$ $x_{i} x_{j}-z_{i} z_{j}$, it is clear from the last line that for any chosen $\mathbf{z}$, $\lim _{\lambda \rightarrow \infty} f(\mathbf{z})$ depends on the values of

$$
L_{\mathbf{x}} \equiv \lim _{\lambda \rightarrow \infty} \exp \left(\lambda \sum_{\mathcal{E}} d_{i j}\right)
$$

for all configurations $\mathbf{x} \in \mathcal{C}$. Considering all possible arrangements of $\left(x_{i}, x_{j}\right)$ and $\left(z_{i}, z_{j}\right)$ and remembering that we have set $\ell=-h$, we find that

$$
d_{i j}=\left\{\begin{array}{cl}
0 & \text { if }\left(x_{i}, x_{j}\right)=\left(z_{i}, z_{j}\right) \text { or }\left(x_{i}, x_{j}\right)=-\left(z_{i}, z_{j}\right) \\
2 h^{2} & \text { if } x_{i}=x_{j} \text { but } z_{i} \neq z_{j} \\
-2 h^{2} & \text { if } x_{i} \neq x_{j} \text { but } z_{i}=z_{j} .
\end{array}\right.
$$

Now choose $\mathbf{z}=\boldsymbol{\ell}$ or $\mathbf{z}=\mathbf{h}$. These are the only two choices for which $z_{i}=z_{j}$ for all edges (because the graph is connected). Then $\sum_{\mathcal{E}} d_{i j} \leq 0$ for every $\mathbf{x}$, with equality only when $\mathbf{x}= \pm \mathbf{z}$. Consequently $L_{\mathbf{x}}=1$ for $\mathbf{x}= \pm \mathbf{z}$ and $L_{\mathbf{x}}=0$ for all other $\mathbf{x}$. From this we conclude that $p_{\mathbf{h}}^{*}$ and $p_{\ell}^{*}$ are as given in the theorem, and therefore all other limiting probabilities must be zero.

The conclusion that the $S_{-h, h}(\boldsymbol{\alpha}, \boldsymbol{\Lambda})$ model is the only variant with more than one positive limiting probability in general may be justified by counterexample. The $n=2$ case (Theorem 3) provides counterexamples for all of the other AL model variants.

Theorem 4 shows that the symmetric models, $S_{-h, h}$, are the only AL/ALR variants that always have reasonable and intuitive large- $\lambda$ behavior.

\subsection{Pseudolikelihood}

Let $\mathbf{z}^{1}, \ldots, \mathbf{z}^{m}$ be a random sample drawn from an autologistic model with $n$ vertices. The pseudolikelihood function is the product of the conditional probabilities,

$$
P L(\boldsymbol{\theta})=\prod_{j=1}^{m} \prod_{i=1}^{n} \operatorname{Pr}\left(Z_{j i}=z_{j i} \mid \mathbf{z}_{-i}^{j}\right)
$$

where $\boldsymbol{\theta}=(\boldsymbol{\alpha}, \lambda)$ is the parameter vector and $z_{j i}$ is the value of the $i$ th variable in the $j$ th observation. Equation (9) is used to compute the conditional probabilities.

Pseudolikelihood is an approximation to the true likelihood [32], and maximum pseudolikelihood (MPL) is widely used as a practical estimation method given the intractability of the partition function for large $n$.

Detailed consideration of the optimization of Equation (21) for all AL and ALR variants is beyond the scope of the present work, but the theorem below and the comments that follow are relevant to comparison of the centered and standard model types. 
THEOREM 5 (pseudolikelihood for standard models). For the standard $A L$ or ALR model with any coding and the simple smoothing assumption, the negative log pseudolikelihood is a convex function of its parameters.

PROOF: The proof is straightforward so most of the details are omitted. Consider the AL model. Note from Equations (8) and (9) that conditional probability for variable $i$ is a function only of parameters $\alpha_{i}$ and $\lambda$. Therefore we can define $q_{j i}\left(\alpha_{i}, \lambda\right) \equiv-\log \left(\operatorname{Pr}\left(Z_{j i}=z_{j i} \mid \mathbf{Z}_{-i}^{j}\right)\right)$ and write the negative $\log$ pseudolikelihood function as

$$
-\log P L(\boldsymbol{\theta})=\sum_{j=1}^{m} \sum_{i=1}^{n} q_{j i}\left(\alpha_{i}, \lambda\right) .
$$

The function is convex if every $q_{j i}(\cdot)$ is a convex function of its parameters. A function of $r$ parameters is convex if it is convex along every line in the parameter space [33], so define $u_{j i}(t)=$ $q_{j i}\left(\alpha_{i 1}+t \alpha_{i 2}, \lambda_{1}+t \lambda_{2}\right)$. Convexity is proven by finding the second derivative of $u_{j i}(t)$ with respect to $t$ and observing that it is positive for any choice of $\alpha_{i 1}, \alpha_{i 2}, \lambda_{1}, \lambda_{2}$, for any coding and for all $i, j$. Convexity for the ALR case follows because the composition of a convex function with a linear one (here, $\alpha_{i}=\mathbf{x}_{i}^{T} \boldsymbol{\beta}$ ) is convex.

This convexity result indicates that obtaining parameter estimates by MPL should be straightforward for any standard variant (including both the traditional zero/one model and the symmetric model). Unfortunately the same can not be said for centered models. The centering term $\mu_{j}$ is a non-convex function of $\alpha_{j}$. Additionally, in the centered model the $i$ th conditional probability is a function of not only $\alpha_{i}$ and $\lambda$, but also of parameters $\alpha_{j}, j \sim i$. Thus we expect more complications with obtaining good parameter estimates even using the simple MPL framework. Indeed, multiple local optima have been observed in the MPL function even for $n=2$ examples, and for larger problems, numerical optimizers may return different MPL parameter estimates depending on the starting point of the search.

\section{NUMERICAL RESULTS}

This section provides numerical examples to complement the theoretical results and shed more light on the differences among the autologistic variants. The first example focuses on better understanding of parameter interpretation in AL models for the $n=2$ case. The second explores the qualitative differences among the variants in a spatial-data regression setting at larger scale $(n=900)$. The third quantifies the distance between the most important ALR variants in a network-structured regression scenario with $n=16$. After these constructed examples, an analysis of the $H$. vulgaris data set is presented.

\subsection{Parameter Interpretation in the Two-Variable Case}

In section 2.5 it was argued that the autologistic model invites a natural interpretation of its parameters as defining a balance between the unary or endogenous part, and the neighborhood effects. To examine whether this interpretation holds for different AL variants, we first restrict our attention to the effects that two variables have on each other.

\subsubsection{Expected Neighbor Effect}

Consider two variables $Z_{i}$ and $Z_{j}$ that may be part of a larger graph. The log odds expression (10) for $Z_{i}$, conditional on its neighbors, involves the neighbor sum $s_{i}$. The contribution of $Z_{j}$ to this sum is $S_{i}=\lambda_{i j}\left(Z_{j}-\mu_{j}\right)$. Its expectation is

$$
E\left[S_{i}\right]=\left\{\begin{array}{cc}
\lambda_{i j} M_{j} & \text { for the } S_{0,1} \text { model } \\
\lambda_{i j}\left(M_{j}-\mu_{j}\right) & \text { for the } \mathrm{C}_{0,1} \text { model } \\
\lambda_{i j}\left(2 M_{j}-1\right) & \text { for the } \mathrm{S}_{-1,1} \text { model }
\end{array},\right.
$$

where $M_{j}$ is the marginal probability $\operatorname{Pr}\left(Z_{j}=h\right)$. It depends on unary parameter $\alpha_{j}$ as well as on $Z_{j}$ 's neighbors and the strength of their association with $Z_{j}$.

The relationships in Equation (22) are shown graphically in Figure 3. For the $S_{0,1}$ model, the expected neighbor effect is always positive-increasing the $\log$ odds that $Z_{i}$ takes the high level-regardless of $M_{j}$. This is another way of expressing the asymmetry inherent in the standard zero/one model. The centered model corrects this problem, in that $E\left[S_{i}\right]$ can take negative values, but the range of possible neighbor effects is now coupled to $\alpha_{j}$, through $\mu_{j}$. This introduces a type of antisymmetry, where larger $\mu_{j}$ values shift the range of possible neighbor effects downward and smaller $\mu_{j}$ values shift it upward. This antisymmetry is the source of the counterintuitive large- $\lambda$ behavior described in Theorem 3.

The symmetric $\mathrm{S}_{-1,1}$ model, by contrast, resolves the asymmetry problem of the zero/one model without introducing extra complexities. The expected neighbor effect varies linearly from $-\lambda_{i j}$ to $\lambda_{i j}$, crossing through zero when $M_{j}=0.5$. This is

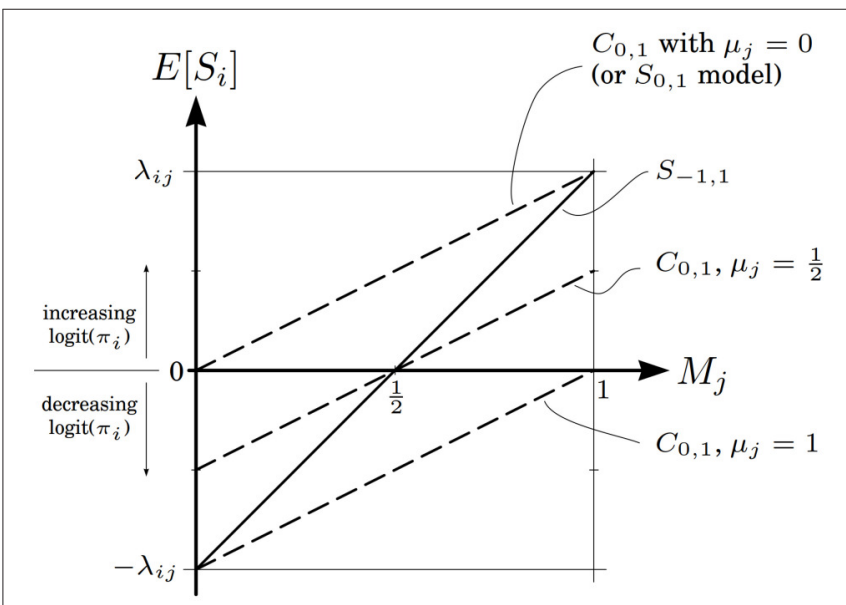

FIGURE 3 | Expected value of the neighbor effect of variable $j$ on variable $i$, as a function of $M_{j}$, the marginal probability that $Z_{j}$ takes the high level. The solid line is for the symmetric model, and dashed lines are for the centered model with different $\mu_{j}$ values. The line for the standard zero/one model coincides with the uppermost dashed line. 
a natural crossing point: when $Z_{j}$ is equally likely to take either state, its average effect on $Z_{i}$ is zero. The centered model only exhibits similar behavior when $\mu_{j}=0.5$.

\subsubsection{Marginal Probability}

Next, consider the $n=2$ case previously encountered in Figure 2. There are two random variables $Z_{1}$ and $Z_{2}$, and three parameters $\alpha_{1}, \alpha_{2}$, and $\lambda$. One way to better understand the parameters' roles is to study how they influence the probability of the event $\left\{Z_{1}=h\right\}$.

It is helpful to consider the effects of the unary parameters not through $\alpha_{1}$ and $\alpha_{2}$ directly, but rather through the endogenous probabilities $p_{1}$ and $p_{2}$, which are monotone functions of them:

$$
p_{i}=\frac{e^{h \alpha_{i}}}{e^{\ell \alpha_{i}}+e^{h \alpha_{i}}}, \quad i=1,2 .
$$

This eliminates a scaling difference that would otherwise confuse the comparison of models with different coding. Note that $p_{i}=$ 0.5 corresponds to $\alpha_{i}=0$.

Figure 4 shows contour plots of $\operatorname{Pr}\left(Z_{1}=h\right)$ as a function of $p_{1}$ and $p_{2}$, for the $S_{0,1}, C_{0,1}$, and $S_{-1,1}$ models. Each row of contour plots in the figure is for a single model variant. The $\lambda$ values for each variant are evenly spaced between 0 and some maximum value. The maximum values were chosen such that $\left|\operatorname{Pr}\left(Z_{1}=h\right)-0.5\right|=0.4$ when $p_{1}=0.5$ and $p_{2}=0.95$ (this is a point at which the neighbor effect of $Z_{2}$ on $Z_{1}$ is large). The $\mathrm{C}_{-1,1}$ model was also plotted but is not shown. Although its maximum $\lambda$ value is different, its contours are identical to those of the $\mathrm{C}_{0,1}$ model.

At $\lambda=0$, the variables are independent and all three variants exhibit the same behavior: $\operatorname{Pr}\left(Z_{1}=h\right)$ increases monotonically with $p_{1}$, unaffected by $p_{2}$. As $\lambda$ increases, we might

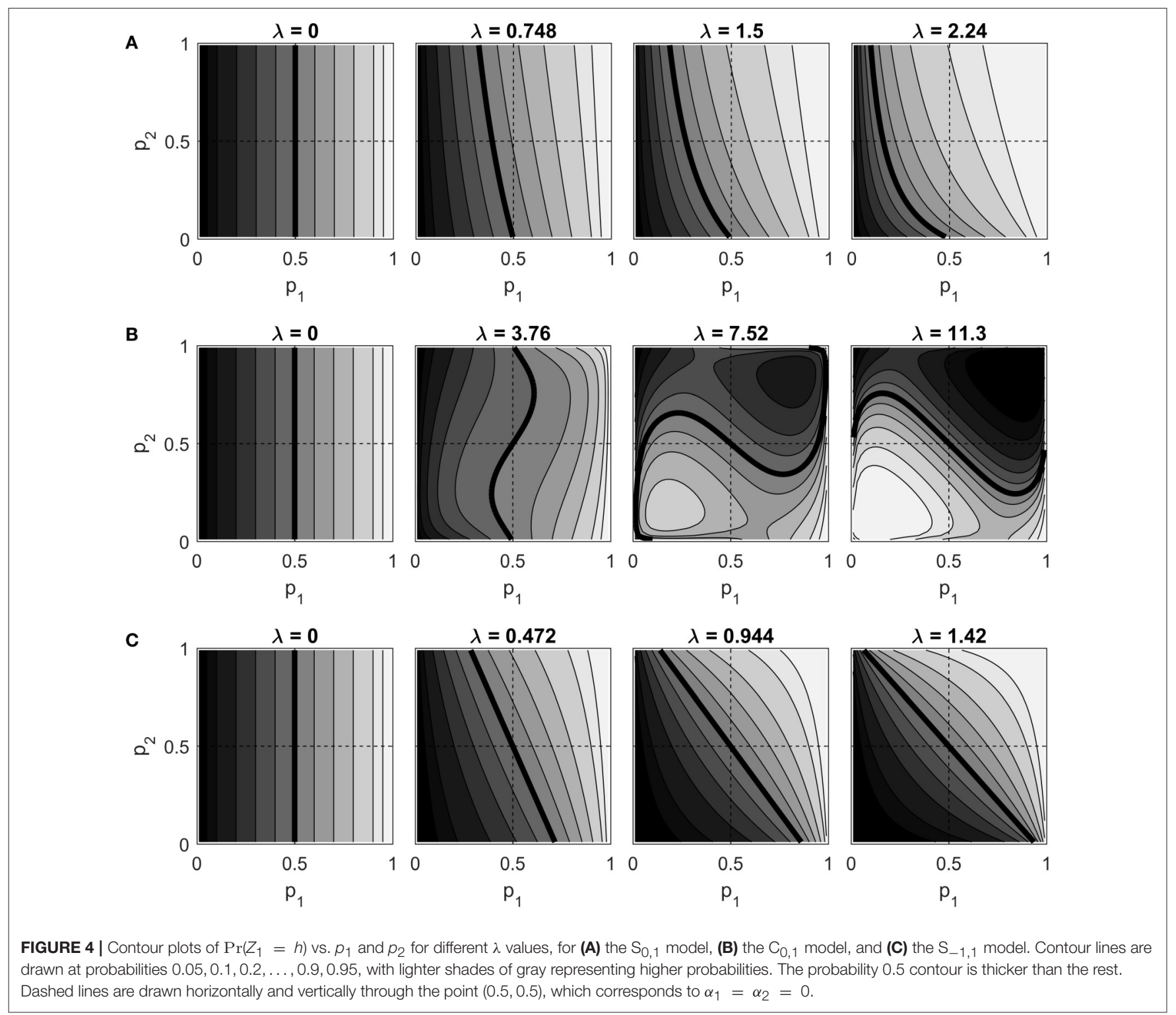


expect (under the natural interpretation) that this probability should remain an increasing function of $p_{1}$, but also become an increasing function of $p_{2}$ as well, due to the neighbor effect. The figure shows that both of the standard models do in fact demonstrate this expected behavior. The $S_{0,1}$ model does so while exhibiting its asymmetry: as $\lambda$ increases, the marginal probability increases throughout the plane, even in areas where both $p_{1}$ and $p_{2}$ are less than one half. The $S_{-1,1}$ model, on the other hand, gives the expected behavior while maintaining a marginal probability of 0.5 at $p_{1}=p_{2}=0.5$ at every choice of $\lambda$.

Turning to the centered model (row B in the figure), it is clear that this model does not show the expected behavior. In this model, increasing $p_{2}$ may either increase or decrease the marginal probability, depending on where one is in the $\left(p_{1}, p_{2}\right)$ plane. While the centered model does maintain $\operatorname{Pr}\left(Z_{1}=h\right)=0.5$ for all $\lambda$ when $p_{1}=p_{2}=0.5$, we again see counterintuitive behavior when $\lambda$ becomes large. The marginal probability that $Z_{1}=h$ is nearly zero in the first quadrant, precisely where both endogenous probabilities are large. Also note that a much larger $\lambda$ value is required to get a strong neighbor effect. This is a direct consequence of the centering adjustment, which subtracts the independence expectation from each $Z_{j}$ in the neighborhood sum.

\subsubsection{Concordance Probability}

Continuing with the same example, we can also consider the probability that $Z_{1}$ and $Z_{2}$ are concordant, that is, $\operatorname{Pr}\left(Z_{1}=\right.$ $Z_{2}$ ). The log odds of this probability are given in Figure 5, as a function of $\lambda$, for the same three variants at different $\left(p_{1}, p_{2}\right)$ combinations. The log odds has been chosen to highlight an interesting difference between the symmetric model and the other variants. For a symmetric model, the odds of event $\left\{Z_{1}=\right.$ $\left.Z_{2}\right\}$ are

$$
\frac{\operatorname{Pr}\left(Z_{1}=Z_{2}\right)}{\operatorname{Pr}\left(Z_{1} \neq Z_{2}\right)}=e^{2 \lambda h^{2}} \frac{e^{2 h\left(\alpha_{1}+\alpha_{2}\right)}+1}{e^{2 h \alpha_{1}}+e^{2 h \alpha_{2}}},
$$

which factorizes into a part depending on $\lambda$ alone and another depending on $\alpha$ alone. As a result the log odds of the event, for any fixed $\left(p_{1}, p_{2}\right)$, is a linear function of $\lambda$ with slope $2 h^{2}$. This invites a simple interpretation of $\lambda$ as an association parameter: a unit change of $\lambda$ will increase the log odds of the two variables taking the same state by $2 h^{2}$, regardless of the values of the unary parameters. This is visible in row $(\mathrm{C})$ of the figure.

The $\mathrm{S}_{0,1}$ model and the centered variants (rows $\mathrm{A}$ and $\mathrm{B}$ ) do not admit such an interpretation. For these two models the log odds curves show nonlinearities that change depending on the values of $p_{1}$ and $p_{2}$. In both cases the curves are difficult to explain intuitively. In row (A), we see that the curves for

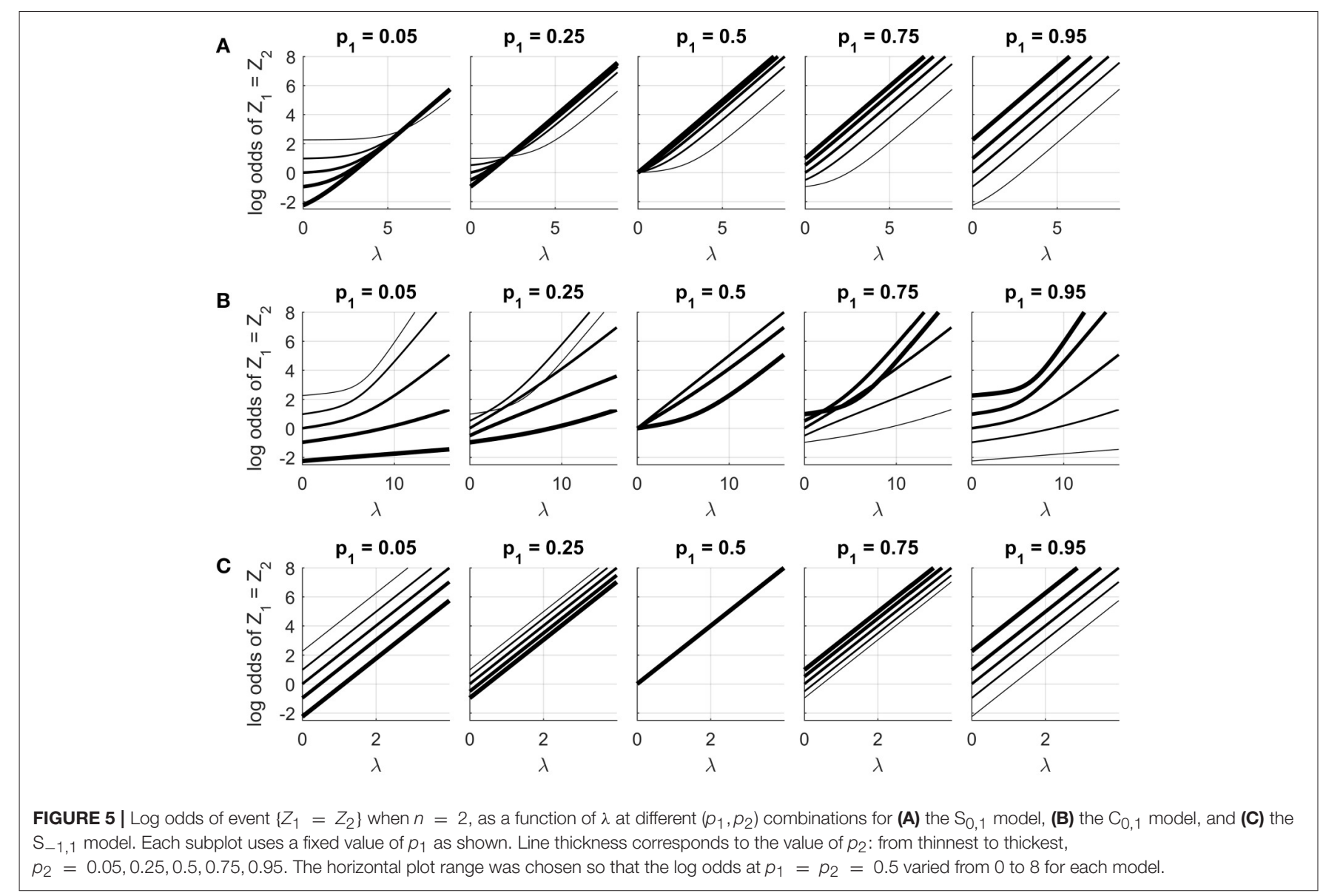


$\left(p_{1}=0.05, p_{2}=0.05\right)$ are not the same as the curves for $\left(p_{1}=0.95, p_{2}=0.95\right)$. This is hard to justify given that the two cases differ only by the labeling of the high and low states. In row (B) we see that the shapes of the curves depend in a complex way on $p_{1}$ and $p_{2}$. For example, the initial slope of the curve is greatest at $\left(p_{1}=0.5, p_{2}=0.5\right)$, and much smaller when $\left(p_{1}=0.95, p_{2}=0.95\right)$. This implies that increasing $\lambda$ from zero will more strongly influence variables that are both indifferent about their state than it will variables that are both strongly biased to the same state.

\subsection{A Spatial Regression Example}

Now consider a larger example with a regression component. Let $\mathrm{Z}$ be a vector of $n=900$ binary variables, jointly distributed according to an ALR model. The graph structure for the model is a regular $30 \times 30$ lattice; each interior node has four neighbors.

The graph is positioned in space such that it covers the unit square, with the lower left vertex at $(0,0)$ and the upper right one at $(1,1)$. Let the spatial coordinates of variable $i$ be $\left(x_{i 1}, x_{i 2}\right)$. For simplicity, let these spatial coordinates (plus an intercept term) be the predictor variables for regression. Defining $\mathbf{X}$ to be a $900 \times 3$ matrix of covariates, with $i$ th row $\mathbf{x}_{i}^{T}=\left(1, x_{i 1}, x_{i 2}\right)$, the $i$ th variable's unary term becomes

$$
\alpha_{i} \equiv \mathbf{x}_{i}^{T} \boldsymbol{\beta}=\beta_{0}+\beta_{1} x_{i 1}+\beta_{2} x_{i 2} .
$$

For this example, we fix $\boldsymbol{\beta}=(-2,2,2)^{T}$ throughout and explore the effect of $\lambda$ on three variants: $\mathrm{S}_{0,1}(\mathbf{X} \boldsymbol{\beta}, \lambda \mathbf{A}), \mathrm{C}_{0,1}(\mathbf{X} \boldsymbol{\beta}, \lambda \mathbf{A})$, and $\mathrm{S}_{-1 / 2,1 / 2}(\mathbf{X} \boldsymbol{\beta}, \lambda \mathbf{A})$. The symmetric model uses $\{-1 / 2,1 / 2\}$ coding to ensure that when $\lambda=0$, it is equivalent to variants coded $\{0,1\}$.

The present example is very similar to the simulation setting used by both Caragea and Kaiser [24] and Hughes et al. [25]. When $\lambda=0$, every variant gives the same logistic regression model with endogenous probabilities varying smoothly over the unit square, from approximately 0.12 in the lower left corner to about 0.88 in the upper right. The diagonal separating these two corners has endogenous probability 0.5. Figure 6 shows these probabilities as a grayscale image. Such displays are referred to as marginal probability maps; by convention they will plot $\operatorname{Pr}\left(Z_{i}=\right.$ $h$ ) and use lighter shades of gray to represent higher probability. The graph corresponding to $\mathbf{Z}$ is also shown on the figure.

For each model, 500 random samples were drawn at each of ten $\lambda$ values equally spaced from 0 to 2.5 . Estimates of the marginal probabilities were obtained from the samples by counting the proportion of draws for which each variable took its high level. Random draws were obtained by perfect sampling, which is relatively straightforward to implement for AL/ALR models (see [25], and references therein; also [34]).

Figure 7 shows the configurations of two random samples, as well as the estimated marginal probability maps, for each model at each $\lambda$. The differences among the models are clear, and agree with the observations in the $n=2$ case. In the standard $\{0,1\}$ model, increasing $\lambda$ increases the chance of observing the high state, at every vertex, regardless of covariate values. In the centered model, smaller $\lambda$ values (up to about 0.75 ) have only a minor effect on the probability map. At larger $\lambda$ values more clustering of the high and low states is visible, but the low states

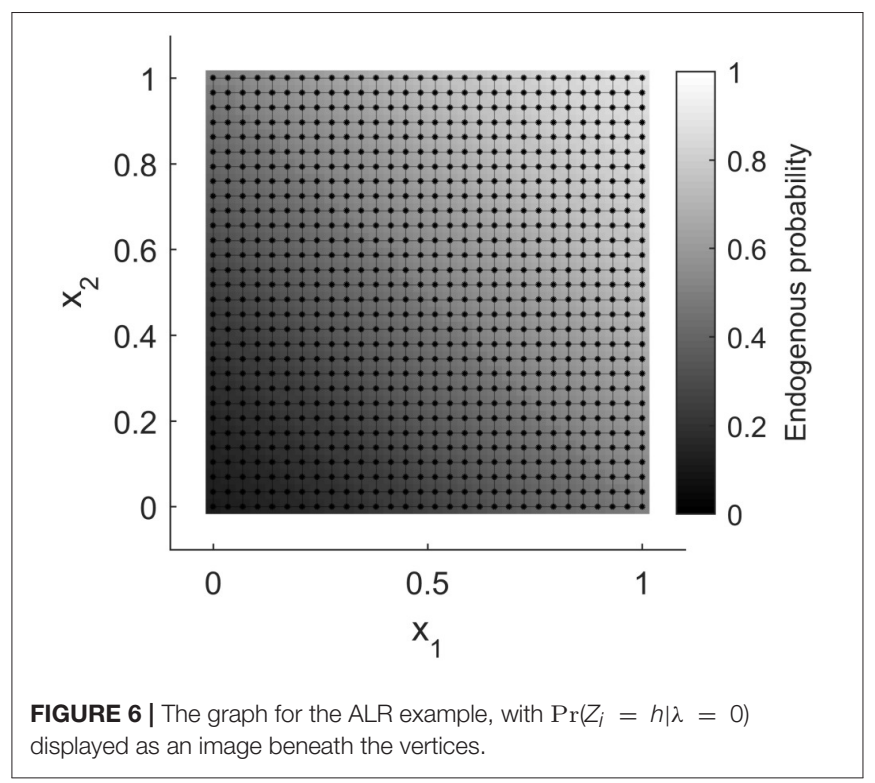

occur at vertices where the endogenous probability is high (and vice versa). For the symmetric model, increasing $\lambda$ causes the probability map to gradually separate around the diagonal, with (low, high) states occurring where the endogenous probability is (low, high).

To more directly assess the effect of $\lambda$ on neighbor interactions, we can consider not just the vertices of the graph but the edges. For any group of edges, the probability of edge concordance can be estimated by the proportion of times those edges were concordant over the 500 random samples. To see how the endogenous probabilities modulate the effect of $\lambda$ on concordance, partition the graph into two subgraphs: $\mathcal{G}_{1}=$ $\left\{Z_{i}: p_{i} \leq \frac{1}{3}\right.$ or $\left.p_{i} \geq \frac{2}{3}\right\}$ and $\mathcal{G}_{2}=\left\{Z_{i}: \frac{1}{3}<p_{i}<\frac{2}{3}\right\}$. Subgraph $\mathcal{G}_{1}$ includes edges in the lower left and upper right of the unit square, where both endogenous probabilities are strongly biased toward one state or the other. Subgraph $\mathcal{G}_{2}$ includes edges near the diagonal, where the average endogenous probability of the edge is not far from one half.

Figure 8 shows the estimated concordance probability of edges in $\mathcal{G}_{1}$ and $\mathcal{G}_{2}$, for the $\mathrm{C}_{0,1}$ and $S_{-1 / 2,1 / 2}$ models. In the symmetric model, the effect of $\lambda$ is roughly the same for edges in either group. For the centered model, increasing the association parameter has a fairly strong effect on $\mathcal{G}_{2}$ but a very small effect on $\mathcal{G}_{1}$. This effect can also be observed by careful inspection of Figure 7 for $\lambda \leq 1$. For the centered model, the smoothing effect of $\lambda$ is focused on the parts of the graph where $p_{i}+p_{j}$ is close to one. This is consistent with the results previously seen in Figure 5.

\subsection{A Network Regression Example}

The preceding two examples have focused on parameter interpretation, but it is not always essential to have a readily interpretable model. In statistical learning applications, for example, out-of-sample predictive accuracy may be the dominant objective. In such a case, there is little reason to be concerned about which variant is used, as long each variant's parameters can 

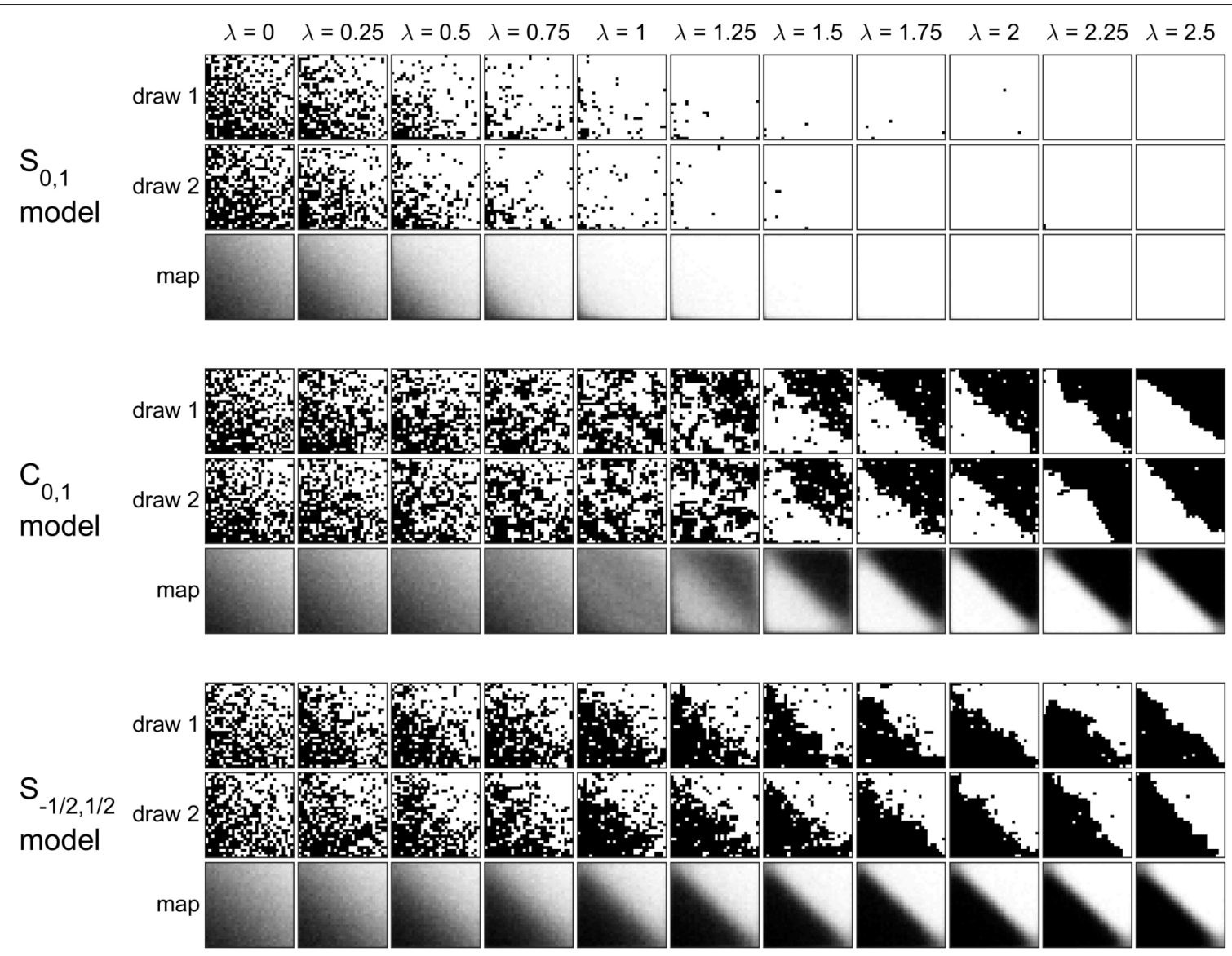

FIGURE 7 | Effect of $\lambda$ on three model variants when $\beta=(-2,2,2)^{T}$. Two randomly-drawn configurations are shown for each model at each $\lambda$ value, along with the estimated marginal probability map. The realizations are displayed as images with black and white pixels representing the low and high states, respectively.

be changed to produce nearly equivalent predictive models. To see whether any concern is justified, we must address the question of how far apart two distribution families defined by ALR models can be.

The approach taken here is to let the symmetric model be the reference distribution family. Instances of this model were generated with random graphs, random covariates, and fixed parameters. Each such instance formed the baseline or target model for a single experimental run. Numerical optimization was then used to find the parameter settings of the $S_{0,1}$ and $C_{0,1}$ models that minimize a measure of statistical distance between them and the target model.

Take the Hellinger distance as our distance measure. If $M_{1}$ and $M_{2}$ are two ALR models with $n$ vertices, which respectively assign probabilities $\mathbf{w}=\left(w_{1}, w_{2}, \ldots w_{2^{n}}\right)^{T}$ and $\mathbf{v}=\left(v_{1}, v_{2}, \ldots v_{2^{n}}\right)^{T}$ to the $2^{n}$ possible configurations, the Hellinger distance between the models is $\frac{1}{\sqrt{2}}\|\sqrt{\mathbf{w}}-\sqrt{\mathbf{v}}\|_{2}$, where the square roots inside the norm are taken componentwise. It varies between zero (when the models are equivalent) and one (when the sets of configurations given positive probability by the models are disjoint). Models that have Hellinger distance not close to zero can not be considered reasonable approximations of one another as predictive models.
For this example we consider graphs and covariates that have a network structure, rather than a spatially-referenced one. Graphs were generated according to a preferential attachment scheme $[35,36]$. For each graph, an initial set of $m_{0}$ fully connected vertices is first created, and additional vertices are added sequentially until there are $n$ nodes in total. Each new node is connected by edges to $m$ existing nodes, selected randomly with weights that are proportional to their degree. In this experiment we consider two cases. Case 1 has $m_{0}=4$ and $m=1$. In this case the graph is structured as tree branches connected to the initial fully-connected four nodes. Case 2 has $m_{0}=m=2$. This case has more connectivity throughout the graph.

The baseline models were generated as follows. Fix $n=$ 16 , which is sufficiently small to allow direct calculation of the Hellinger distance. Set $\lambda=0.25$, and generate 200 graphs for each of Case 1 and Case 2. For each graph, assign to each vertex a linear predictor $\alpha_{i}=\beta_{0}+\beta_{1} x_{i}$, where $x_{i}, i=1 \ldots 16$ are drawn independently from a standard normal distribution. Each graph then has its own adjacency matrix $\mathbf{A}$ and covariate matrix $\mathbf{X}$; take the $S_{-1,1}(\mathbf{X} \boldsymbol{\beta}, \lambda \mathbf{A})$ model with $\beta_{0}=0$ and $\beta_{1}=1$ as the target model corresponding to that graph. Repeat this process for $\lambda=0.5,0.75,1.0,1.25$, and 1.5 . 


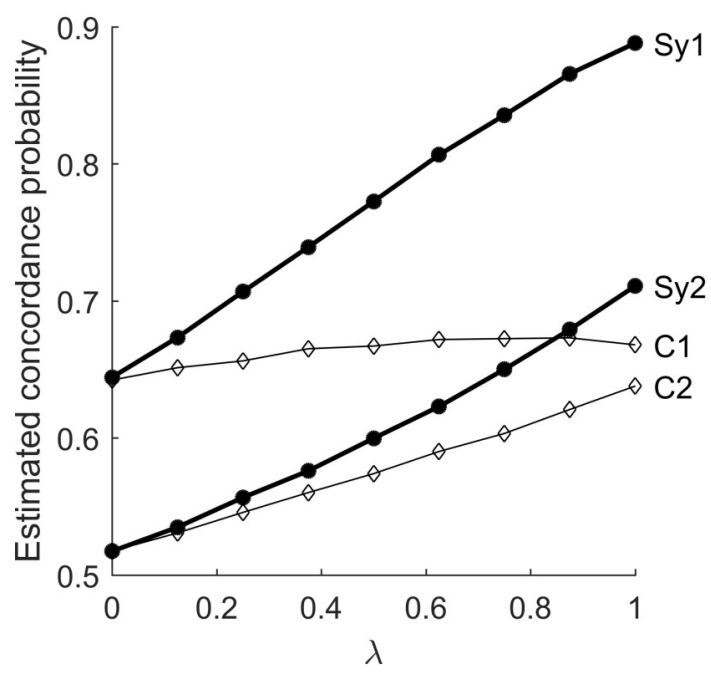

FIGURE 8 | Estimated concordance probability of edges in subgraphs $\mathcal{G}_{1}$ and $\mathcal{G}_{2}$, vs. $\lambda$. C1 and $\mathrm{C} 2$ are the results for subgraphs 1 and 2 , respectively, using the centered zero/one model. Sy1 and Sy2 are the corresponding curves for the symmetric model.

Figure 9 gives examples of graphs generated from the two cases. The baseline models correspond to a situation where the endogenous probabilities of neighbor variables are noisy and uncorrelated. Models with larger $\lambda$ values will put higher probability on configurations that have clusters of high or low values, with cluster size and location modulated by the endogenous probabilities. In the limit as $\lambda \rightarrow \infty$, the two saturated states $\mathbf{Z}=-\mathbf{1}$ and $\mathbf{Z}=\mathbf{1}$ will get all of the probability mass, according to Equation (20).

Numerical optimization was performed to find models $\mathrm{S}_{0,1}(\mathbf{X} \hat{\boldsymbol{\beta}}, \hat{\lambda} \mathbf{A})$ and $\mathrm{C}_{0,1}(\mathbf{X} \tilde{\boldsymbol{\beta}}, \tilde{\lambda} \mathbf{A})$ that minimize the Hellinger distance to each target. Figure 10A gives violin plots [37] of the distances. Each "violin" in the plot is based on an unequallyspaced histogram with bin edges at the sample quintiles. The median value is indicated by a dot.

The figure reveals striking differences between the nearest models. All but three of the experimental settings produce median distances greater than 0.2 , and many are greater than 0.4. This means that the $\mathrm{S}_{0,1}$ and $\mathrm{C}_{0,1}$ models cannot apportion probability mass to configurations in a manner very similar to the symmetric model. The distances also have high variability. This indicates that the ability of the standard and centered variants to approximate the symmetric model is strongly influenced by the random aspects of the target model: the graph structure and the covariate values.

The distribution of distances for the standard zero/one model becomes bimodal as $\lambda$ gets large. Inspection of the data showed a strong relationship between the sum of the covariates and the Hellinger distance. Larger negative values of $\sum x_{i}$ corresponded to larger distances. In this situation, the target model puts more weight on configurations with many low states, but the $S_{0,1}$ model can not easily do the same, because increasing $\lambda$ always promotes the high state.
The distributions of the centered models' distances are not bimodal, but they also exhibit considerable variation. For Case 2 target models, the median Hellinger distance of the $\mathrm{C}_{0,1}$ model begins to decrease sharply when the target model's association parameter is greater than one half. One reason for this is that the probability mass of the target distribution begins to concentrate around a small number of configurations near the saturated $\ell$ and $\mathbf{h}$ states, essentially making it easier to approximate the distribution. This effect is stronger in Case 2 than in Case 1 , because the higher connectivity of the graph in Case 2 strengthens the neighborhood effect at a given $\lambda$ level.

This example demonstrates that we can not treat the different ALR variants as interchangeable, even approximately, and even if we are only concerned with predictive modeling. If we are also interested in parameter interpretation, then the regression parameter estimates are of particular interest, since they always define the endogenous probabilities. To compare the linear predictors of the models, we can calculate the average absolute difference of the 16 endogenous probabilities, relative to the target model. Figure 10B shows the distribution of these averages for the experimental runs. For the centered model, we see that when the Hellinger distances are small, the endogenous differences are large. This shows that when $\lambda>0$, we can not find a situation where the centered and symmetric models provide similar probability distributions and similar interpretations at the same time.

\subsection{Analysis of the Hydrocotyle Vulgaris Data}

We now return to the $H$. vulgaris data to see how the differences among the ALR variants can manifest themselves in a real application. The $\mathrm{S}_{0,1}, \mathrm{C}_{0,1}$, and $\mathrm{S}_{-1 / 2,1 / 2}$ models were all fit to the data using a four-nearest-neighbor graph and the MPL method. Optimization of the pseudolikelihood was attempted from multiple random starting points. For the two standard models, the same solution was found each time (unsurprisingly, given Theorem 5). For the centered variant, three locally-optimal solutions were found; the one with greatest log pseudolikelihood was assumed to be the global optimum. Let $\beta_{0}, \beta_{1}$, and $\lambda$ be the intercept, the coefficient of altitude, and the association parameter, respectively.

Results of the analysis are shown in Figure 11, which shows the estimated marginal probability maps, and Table 1, which gives the coefficient estimates. The table includes standard errors obtained by the parallel parametric bootstrap as in Hughes et al. [25]. A column labeled "impact" is also given for each parameter. This column holds the covariate impact, as defined in Bardos et al. [23]. In that work, the authors observed a covariate amplification/parameter attenuation effect in auto-models, where (relative to logistic regression) smaller coefficient magnitudes were required to obtain similar observed effects on the fitted values. The impact is an alternative measure of a covariate's influence on the response, intended to be informative despite structural differences across models. The impact is obtained by setting the coefficient in question to zero and re-evaluating the marginal 

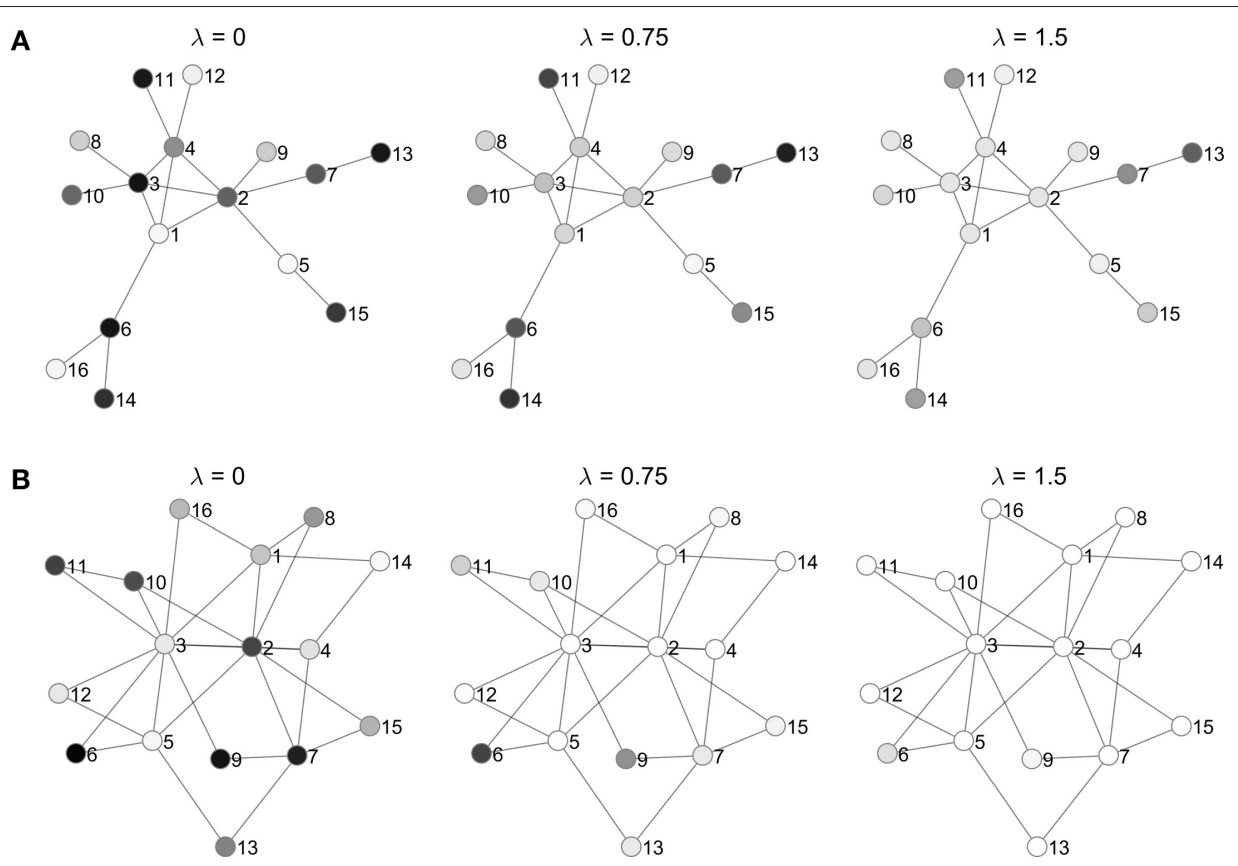

FIGURE 9 | Examples of symmetric ALR models obtained by randomly generating a graph and covariates according to (A) Case 1 and (B) Case 2 as described in the text. Each model is shown for three $\lambda$ values, to illustrate the neighborhood effect. Nodes are shaded to indicate their marginal probability of taking the high level (lighter shades indicate higher probability).

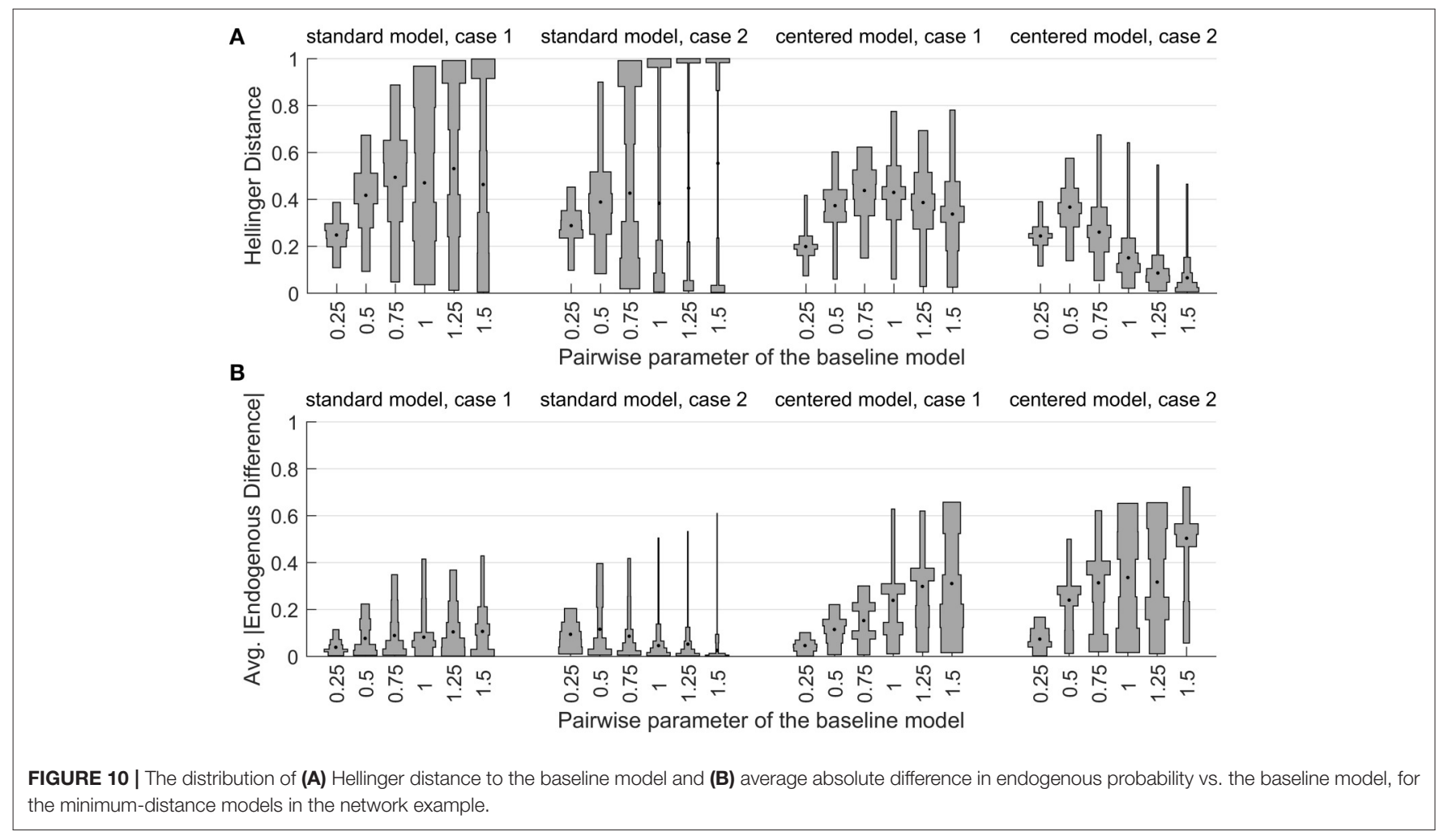

probabilities of the model. The average (across vertices in the graph) of the absolute change in marginal probability is the impact.
If we look only at the magnitudes of $\hat{\lambda}$ and $\hat{\beta}_{1}$, the three ALR models appear quite similar. All have $\hat{\lambda} \approx 1.5$, indicating strong spatial association. All have $\hat{\beta}_{1} \approx-0.15$, which is much 


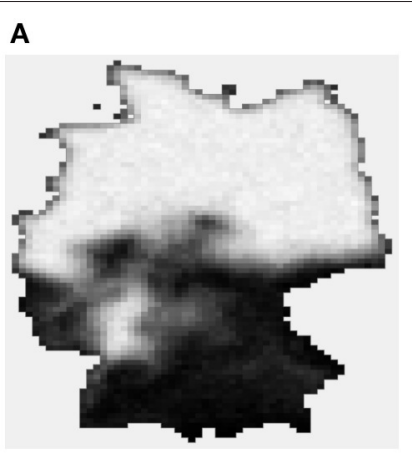

B

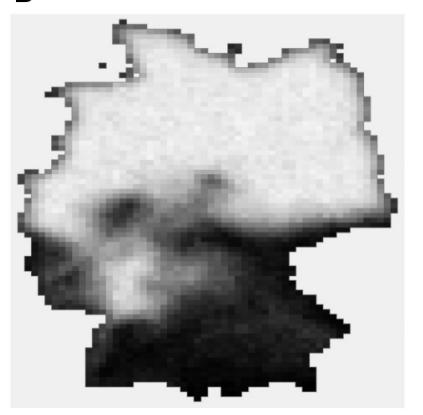

C

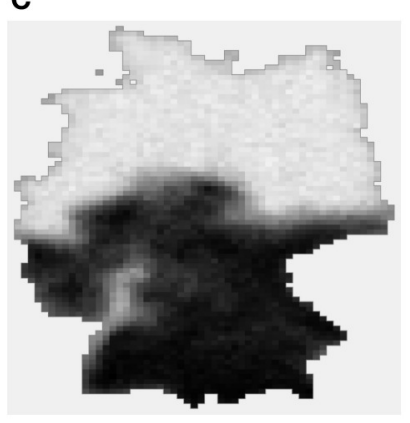

D

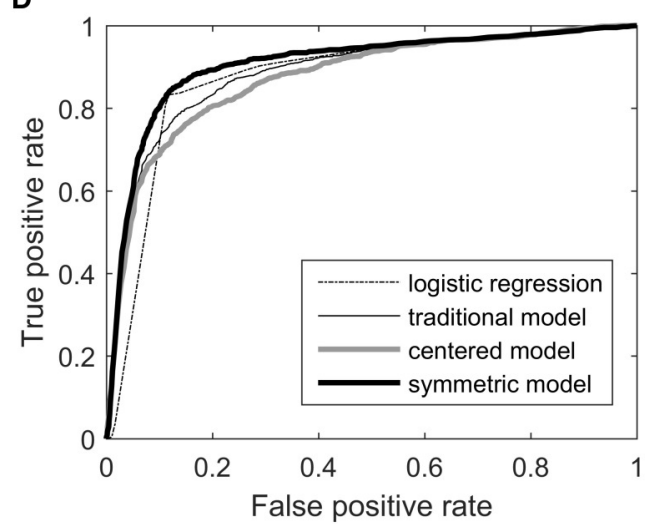

FIGURE 11 | Results of ALR analysis of the H. vulgaris data. The top row shows marginal probability maps for (A) the traditional $\mathrm{S}_{0,1}$ model, (B) the centered model, and (C) the symmetric model. Plot (D) gives the receiver operating characteristic curves for the three models as well as ordinary logistic regression.

TABLE 1 | Parameter estimation results for the H. vulgaris data.

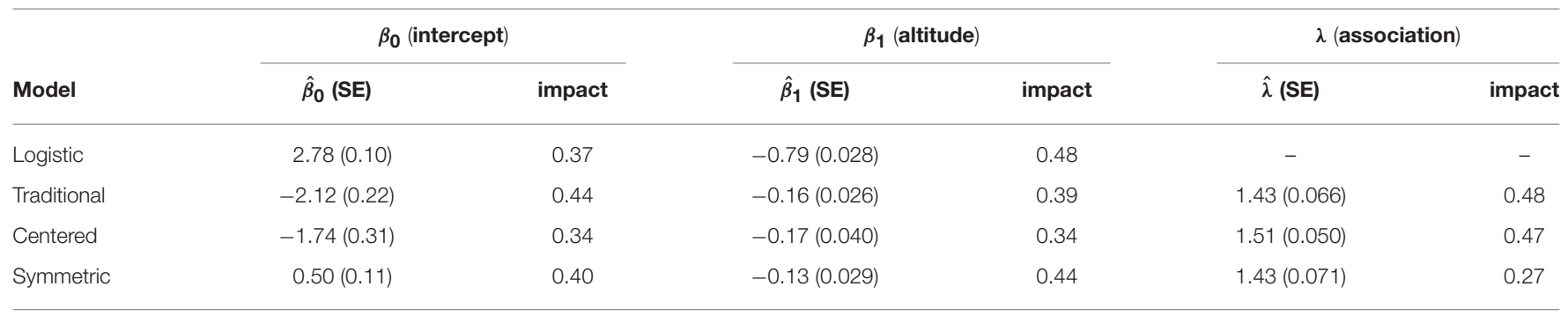

smaller than the logistic regression value of -0.79 (evidence of the parameter attenuation phenomenon).

Interpretation difficulties begin to arise for the $S_{0,1}$ and $C_{0,1}$ models, however, when we consider $\hat{\beta}_{0}$. Both models assign large negative values to the intercept, suggesting that when the altitude is zero everywhere, the species should be largely absent. This is contrary to the observed facts, where species presence has a clear association with low elevation. The coefficient of altitude (a positive quantity) is also negative in both models, leaving no obvious way for species presence to take a high probability. Indeed, the maximum endogenous probability over the entire map for either model is less than 0.2. How is it, then, that the fitted models assign high probability in the northern part of the map?
It is only because $\lambda$ is large enough to distort the meaning of the regression part of the model. This is reflected in the large value of impact for $\lambda$ in these two models. Adding the neighbor effect to either model has a drastic effect on the fitted values everywhere in the map.

Contrast this with the interpretation of the symmetric model. Its coefficients have the same signs as, and magnitudes roughly proportional to, the logistic regression model. The median endogenous probability across the map is 0.49 for logistic regression and 0.51 for the symmetric model. The neighbor effect promotes the marginal probabilities to intensify (toward either zero or one) if local regions' endogenous tendencies agree. As a result the impact of $\lambda$ is much lower than it is for the other 
ALR variants. Compared to the centered model, the regression parameters have much higher impact values and much more precise estimates.

The probability maps support the notion that the symmetric model is more appropriate for these data. Its marginal probabilities more closely match the altitude map and the obeserved pattern of species presence seen in Figure 1. There also appears to be an edge effect in the northern half of map for the traditional and centered models, which is not present in the symmetric case.

A more structured method of comparing goodness-of-fit and potential predictive power is to produce a receiver operating characteristic curve for each model. This was done for the same data by Bardos et al. [23], but only for logistic regression and the traditional $S_{0,1}$ model. In Figure 11D a similar plot is shown with the centered and symmetric models included. The symmetric model dominates the other three models.

\section{DISCUSSION}

The preceding sections provided results about model equivalence and parameter interpretation for autologistic models. They have important implications for analysts considering using these models.

The main results about equivalence apply for models with nonzero association matrices and arbitrary full-rank covariate matrices. They are:

- Every AL model (centered or standard, any coding) is equivalent to any chosen standard AL model.

- Two standard ALR models are equivalent if their codings differ by a positive scaling factor (otherwise they are not).

- Any two centered ALR models with different codings are not equivalent.

- Any given standard ALR model is not equivalent to any given centered ALR model, even if their coding is the same.

It was shown by example that the statistical distance between non-equivalent models can be large, making model choice a matter of practical consequence. Given the known limitations of the $\mathrm{S}_{0,1}$ model, the choice is mainly between the centered $\{0,1\}$ and symmetric $\{-h, h\}$ variants.

\subsection{A Case for the Symmetric Model}

The centered and symmetric models can be thought of as competing alternatives, each aiming to remedy the parameter interpretation problem of the traditional $S_{0,1}$ model.

The centered model modifies the algebraic form of the traditional model. It does so in a manner analogous to Gaussian models, with the goal of making the regression parameters directly control the marginal probabilities. This goal is not achievable for dichotomous variables, however, because the covariance of any two such variables is functionally related to their marginal probabilities. As a result, centering achieves its goal only approximately, and only over a restricted region of the parameter space.
In exchange for this modest benefit, the centered model introduces extra analytical and computational difficulties. One such difficulty is non-convexity of the pseudolikelihood. Another, more critical, one is the counterintuitive behavior of the model when association is strong. This undesirable side effect was not noted in the initial articles about the centered model-perhaps because only $\lambda$ values in the range $[0,1]$ were considered-but it has been subsequently documented [38]. It is not easy to say in advance when it will become problematic for a specific data set and graph structure, but when it does, any interpretation benefit will surely be lost. Users of the centered model must be prepared to check carefully for the problem after parameter estimation.

The preceding sections have demonstrated that the problems with the traditional model are not due to its algebraic form or its lack of centering, but simply due to the asymmetry of the $\{0,1\}$ coding. The symmetric model only changes the coding, but this change is sufficient to allow a very natural interpretation of the parameters. The regression parameters determine the endogenous structure, and $\lambda$ provides a balance between the endogenous structure and the neighbor effect. This favorable interpretation remains the same regardless of the strength of association (and, by Theorem 4, the symmetric variants are unique in this regard). The symmetric model resolves the problems with the traditional model in a simple way, without introducing extra difficulties. Indeed, one could argue that if the symmetric model had been proposed first, there would be little reason to consider the centered model.

The H. vulgaris example demonstrated both the deficiencies of the centered model and the advantages of the symmetric one. Using the centered model, the regression parameters lacked a reasonable interpretation, and this happened without other obvious signs of problems with the model (the estimate of $\lambda$ was not unreasonably large, and there was not dramatic lack of fit). The symmetric model, on the other hand, provided a more natural parameter interpretation with a closer connection to logistic regression. At the same time it had better fit, larger covariate impact, and more precise regression parameter estimates.

The ultimate arbiters of model quality in practice are goodness-of-fit and suitability for modeling objectives. It is not possible to declare in advance that a single variant is preferable in all circumstances. Nevertheless, all of the results of the present work point to the symmetric model as the autologistic variant with the most desirable properties. Future researchers are strongly recommended to consider the symmetric model as the starting point for their AL/ALR analyses, unless the physical data-generating process has clear links to an alternative model.

\subsection{The Symmetric Model with Bernoulli Responses}

The symmetric $S_{-h, h}$ model could be criticized for two minor drawbacks. First, the value of $h$ is not specified. Second, since the coding is not $\{0,1\}$, we can not say that $E\left[Z_{i}\right]=\operatorname{Pr}\left(Z_{i}=1\right)$. 
TABLE 2 | Model formulae for the symmetric ALR model, in two forms.

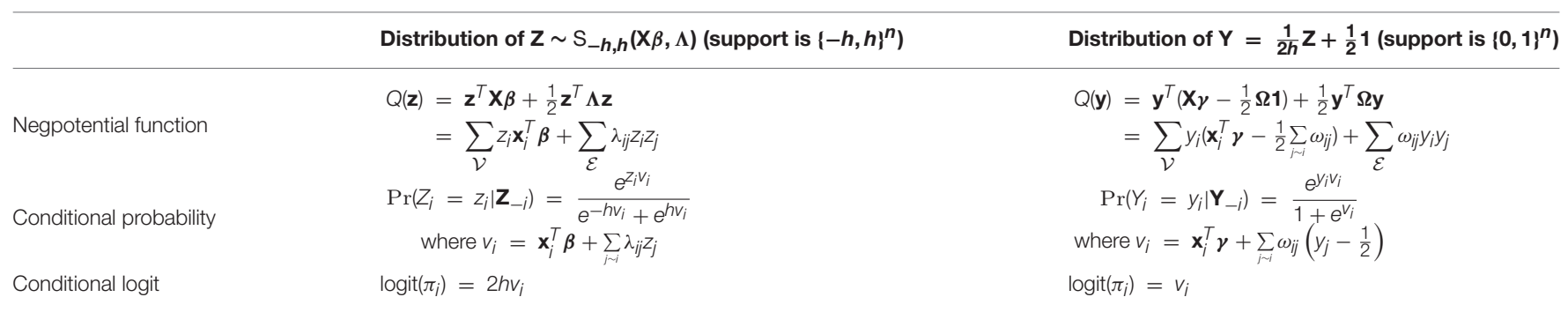

Parameters satisfy $\boldsymbol{\Omega}=4 h^{2} \boldsymbol{\Lambda}$ and $\boldsymbol{\gamma}=2 h \boldsymbol{\beta}$.

The latter drawback denies us the convenience of directly using sample averages to approximate probabilities, as we can do with Bernoulli-distributed random variables.

Both of the drawbacks can be eliminated by performing a transformation to make the symmetric model use the more familiar zero/one binary variables. If random variables $\mathbf{Z}$ are distributed according to the recommended $S_{-h, h}(\mathbf{X} \boldsymbol{\beta}, \boldsymbol{\Lambda})$ model, with $h$ unspecified, transforming $\mathbf{Z}$ into $\mathbf{Y}$ according to

$$
\mathbf{Y}=\frac{1}{2 h} \mathbf{Z}+\frac{1}{2} \mathbf{1}
$$

will leave $f_{\mathbf{Y}}$ equivalent to $f_{\mathbf{Z}}$, but with support $\{0,1\}^{n}$ instead of $\{-h, h\}^{n}$. Note that this is a proper transformation of variables, not a just a change of coding (see Appendix A). The model has not been fundamentally altered.

Table 2 shows the model formulae for $\mathbf{Z}$ and $\mathbf{Y}$ as just described. It may be taken as a summary of the recommended model, in two equivalent forms. The parameters in $f_{\mathrm{Y}}$ have been written as $\boldsymbol{\gamma}=2 h \boldsymbol{\beta}$ and $\boldsymbol{\Omega}=4 h^{2} \boldsymbol{\Lambda}$ to suppress the dependence on $h$, but parameter interpretation is not affected. The $f_{\mathrm{Z}}$ form makes the roles of the parameters most clear, while the $f_{\mathrm{Y}}$ form does not depend on $h$ and shows the model as a natural extension of logistic regression. Since the use of the model as a spatial extension of logistic regression is very common, we may anticipate that $f_{\mathbf{Y}}$, with conditional logit

$$
\operatorname{logit}\left(\pi_{i}\right)=\mathbf{x}_{i}^{T} \boldsymbol{\gamma}+\sum_{j \sim i} \omega_{i j}\left(y_{j}-\frac{1}{2}\right)
$$

and $Y_{i} \in\{0,1\}$, will be the most user-friendly form. It is interesting (but perhaps not surprising, in light of Figure 3) to note that $f_{\mathrm{Y}}$ looks the same as the centered ALR model, but with the centering adjustment equal to $\frac{1}{2} \mathbf{l}$ instead of $\boldsymbol{\mu}_{\boldsymbol{\gamma}}$.

\subsection{Final Remarks}

Having now chosen the symmetric model as a preferred variant, and realized that the ALR variants are not equivalent, it is suitable to begin considering questions of parameter estimation and model performance for the symmetric model, along the lines of Dormann [39] (which used the standard zero/one model) and Hughes et al. [25] (which used the centered zero/one model). Measures of goodness-of-fit should be a significant part of any such studies. Further investigation along this line is planned for the future.

While it is true that different ALR variants are non-nested models, the general form of section 2.3 does link the variants as part of a larger family. This suggests the idea of estimating the coding along with the other parameters. One could, for example, consider only the standard variants, let $\ell=-1$ (for identifiability) and then estimate $h$ from the data. This is somewhat of a technical curiosity, since it is hard to imagine what meaning one would assign to $h$; but it would make the model more flexible.

It would be of considerable practical interest to extend the ALR model beyond the simple smoothing assumption to an adaptive smoothing model, where the pairwise parameter is a function of the neighbor variables' states (and possibly covariates). This is also known as a metric MRF approach [30]. The standard model with plus/minus coding should again be best suited to this extension, which would greatly increase model flexibility. Initial exploration of this approach has shown promise.

The autologistic model is a pairwise MRF model; it is possible to construct MRFs for binary responses where cliques of more than two variables contribute to the negpotential function. In this more general case, the crucial aspect of model construction is to design the clique potential functions ( $\psi_{m}$ in Equation 2 ) in a way that reflects the model's intended purpose. Being aware of the potential impact of coding changes, it would be wise to construct the clique potentials in a way that is invariant to the coding. If this is not possible, the role of coding should be considered carefully to determine the best choice.

Another extension of the AL/ALR models is to the case of categorical responses with more than two levels. Again, the crucial task is the appropriate design of the clique potentials. When there are three or more levels, dependence on the coding is likely to increase in complexity. The multilevel logistic model $[7,21]$ is one extension of the $\mathrm{AL}$ model to handle more than two levels. It overcomes the coding problem by simply checking whether every variable in a clique takes the same value. If all of the values are equal, the clique potential is +1 ; otherwise, it is -1 . The result is a negpotential function very similar to that of the symmetric AL model. Further development of models of this type, including a regression component and a clear connection to standard multinomial 
logistic (softmax) regression, is a potentially fruitful avenue for future work.

Finally, it is possible that the simple observation behind this work-that even a linear transformation of response coding may be a non-trivial operation-could have implications in other use cases as well. Probabilistic graphical models find application in a variety of pattern recognition and deep learning architectures, which are continually being extended in various directions. It is advisable to pay attention to variable coding, to determine if it can change the nature of the model, or be exploited to improve performance.

\section{REFERENCES}

1. Besag JE. Nearest-neighbour systems and the auto-logistic model for binary data. J R Stat Soc Ser B (1972) 34:75-83.

2. Besag J. Spatial interaction and the statistical analysis of lattice systems. $J R$ Stat Soc Ser B (1974) 36:192-236.

3. Kaiser MS, Cressie N. The construction of multivariate distributions from Markov random fields. J Multiv Anal. (2000) 73:199-220. doi: 10.1006/jmva.1999.1878

4. Baxter RJ. Exactly Solved Models in Statistical Mechanics. London, UK: Academic Press Limited (1982).

5. Cipra BA. An introduction to the ising model. Am Math Monthly (1987) 94:937-59. doi: 10.2307/2322600

6. Geman S, Graffigne C. Markov random field image models and their applications to computer vision. In: Proceedings of the International Congress of Mathematicians. Vol. 1. Berkeley, CA (1986). p. 2.

7. Li SZ. Markov Random Field Modeling in Image Analysis. London, UK: Springer (2009).

8. Blake A, Kohli P, Rother C, editors. Markov Random Fields for Vision and Image Processing. Cambridge, MA: MIT Press (2011).

9. Hinton GE. Boltzmann machine. Scholarpedia (2007) 2:8. doi: 10.4249 /scholarpedia.1668

10. Cox DR. The analysis of multivariate binary data. J R Stat Soc Ser C (1972) 21:113-20. doi: 10.2307/2346482

11. Zhao LP, Prentice RL. Correlated binary regression using a quadratic exponential model. Biometrika (1990) 77:642-8. doi: 10.1093/biomet/77. 3.642

12. Cox DR, Wermuth N. A note on the quadratic exponential binary distribution. Biometrika (1994) 81:403-8. doi: 10.1093/biomet/81.2.403

13. Dai B, Ding S, Wahba G. Multivariate bernoulli distribution. Bernoulli (2013) 19:1465-83. doi: 10.3150/12-BEJSP10

14. $\mathrm{Wu} \mathrm{H}$, Huffer FRW. Modelling the distribution of plant species using the autologistic regression model. Environ Ecol Stat. (1997) 4:31-48. doi: 10.1023/A:1018553807765

15. He F, Zhou J, Zhu H. Autologistic regression model for the distribution of vegetation. J Agricul Biol Environ Stat. (2003) 8:205-22. doi: 10.1198/1085711031508

16. Kirkham J, Kaur R, Stillman EC, Blackwell PG, Elcock C, Brook AH. The patterning of hypodontia in a group of young adults in Sheffield, UK. Arch Oral Biol. (2005) 50:287-91. doi: 10.1016/j.archoralbio.2004.11.015

17. Bandyopadhyay D, Reich BJ, Slate EH. Bayesian modeling of multivariate spatial binary data with applications to dental caries. Stat Med. (2009) 28:3492-508. doi: 10.1002/sim.3647

18. Towner MC, Grote MN, Venti J, Mulder MB. Cultural macroevolution on neighbor graphs. Hum Nat. (2012) 23:283-305. doi: 10.1007/s12110-012-9142-Z

19. Zhang N, Yang Q. A random effect autologistic regression model with application to the characterization of multiple microstructure samples. IIE Trans. (2016) 48:34-42. doi: 10.1080/0740817X.2015.1047069

20. Kumar S, Hebert M. Discriminative random fields. Int J Comput Vis. (2006) 68:179-201. doi: 10.1007/s11263-006-7007-9

21. Li J, Bioucas-Dias JM, Plaza A. Spectral-spatial hyperspectral image segmentation using subspace multinomial logistic regression and Markov

\section{AUTHOR CONTRIBUTIONS}

The author confirms being the sole contributor of this work and approved it for publication.

\section{ACKNOWLEDGMENTS}

The author would like to thank Charmaine Dean for providing the application that led to the present work, and for helpful comments on a draft of this manuscript.

random fields. IEEE Trans Geosci Remote Sens. (2012) 50:809-23. doi: 10.1109/TGRS.2011.2162649

22. Carl G, Kühn I. Analyzing spatial autocorrelation in species distributions using Gaussian and logit models. Ecol Model. (2007) 207:159-70. doi: 10.1016/j.ecolmodel.2007.04.024

23. Bardos DC, Guillera-Arroita G, Wintle BA. Covariate influence in spatially autocorrelated occupancy and abundance data. arXiv:1501.06530v2 (2015).

24. Caragea PC, Kaiser MS. Autologistic models with interpretable parameters. J Agricult Biol Environ Stat. (2009) 14:281-300. doi: 10.1198/jabes.2009.07032

25. Hughes J, Haran M, Caragea PC. Autologistic models for binary data on a lattice. Environmetrics (2011) 22:857-71. doi: 10.1002/env.1102

26. Besag J. On the statistical analysis of dirty pictures. J R Stat Soc Ser B (1986) 48:259-302.

27. Kindermann R, Snell JL. Markov Random Fields and Their Applications. Providence, RI: American Mathematical Society (1980).

28. Rue H, Held L. Gaussian Markov Random Fields. Boca Raton, FL: Chapman \& Chap/CRC (2005).

29. Bishop CM. Pattern Recognition and Machine Learning. New York, NY: Springer (2006).

30. Koller D, Friedman N. Probabilistic Graphical Models. Cambridge, MA: The MIT Press (2009).

31. Adams RA, Essex C. Calculus: Several Variables, 7th Edn. Toronto, ON: Pearson Education Canada (2010).

32. Besag J. Statistical analysis of non-lattice data. J R Stat Soc Ser D (The Statistician) (1975) 24:179-95. doi: 10.2307/2987782

33. Boyd S. Convex Optimization. Cambridge, UK: Cambridge University Press (2004).

34. Fox C, Nicholls GK. Exact MAP states and expectations from perfect sampling: greig, porteous and seheult revisited. In: Bayesian Inference and Maximum Entropy Methods in Science and Engineering: 20th International Workshop. Vol. 568. Gif-sur-Yvette (2001). p. 252.

35. Barabási AL, Albert R. Emergence of scaling in random networks. Science (1999) 286:509-12. doi: 10.1126/science.286.5439.509

36. Albert R, Barabási AL. Statistical mechanics of complex networks. Rev Mod Phys. (2002) 74:47-97. doi: 10.1103/RevModPhys.74.47

37. Hintze JL, Nelson RD. Violin plots: a box plot-density trace synergism. Am Stat. (1998) 52:181-4.

38. Kaiser MS, Caragea PC, Furukawa K. Centered parameterizations and dependence limitations in Markov random field models. J Stat Plan Inference (2012) 142:1855-63. doi: 10.1016/j.jspi.2012.02.030

39. Dormann CF. Assessing the validity of autologistic regression. Ecol Model. (2007) 207:234-42. doi: 10.1016/j.ecolmodel.2007.05.002

Conflict of Interest Statement: The author declares that the research was conducted in the absence of any commercial or financial relationships that could be construed as a potential conflict of interest.

Copyright (C) 2017 Wolters. This is an open-access article distributed under the terms of the Creative Commons Attribution License (CC BY). The use, distribution or reproduction in other forums is permitted, provided the original author(s) or licensor are credited and that the original publication in this journal is cited, in accordance with accepted academic practice. No use, distribution or reproduction is permitted which does not comply with these terms. 


\section{APPENDIX}

\section{A. WHAT IT MEANS TO CHANGE THE CODING OF A MODEL}

The distinction between transforming a random variable to a new coding and changing the coding of a model will now be clarified. Suppose that $\mathbf{Z}$ is a binary variable with any coding, having PMF with parameter vector $\boldsymbol{\theta}$ :

$$
f_{\mathbf{Z}}(\mathbf{z})=\operatorname{Pr}(\mathbf{Z}=\mathbf{z}) \propto g(\mathbf{z} ; \boldsymbol{\theta}) .
$$

Transformation of variables can be viewed as the correct way to alter a model to handle a different coding. A random variable with any desired coding, having distribution equivalent to $f_{\mathbf{Z}}$, can be obtained by the one-to-one transformation

$$
\mathbf{Y}=a \mathbf{Z}+b \mathbf{1} \Longleftrightarrow \mathbf{Z}=\frac{1}{a} \mathbf{Y}-\frac{b}{a} \mathbf{1}
$$

with appropriate choices of $a$ and $b$. The PMF of $\mathbf{Y}$ is

$$
\begin{aligned}
f_{\mathbf{Y}}(\mathbf{y}) & =\operatorname{Pr}(\mathbf{Y}=\mathbf{y})=\operatorname{Pr}(a \mathbf{Z}+b \mathbf{1}=\mathbf{y})=f_{\mathbf{Z}}\left(\frac{1}{a} \mathbf{y}-\frac{b}{a} \mathbf{1}\right) \\
& \propto g\left(\frac{1}{a} \mathbf{y}-\frac{b}{a} \mathbf{1} ; \boldsymbol{\theta}\right)=g(\mathbf{z} ; \boldsymbol{\theta}) .
\end{aligned}
$$

In this sense we can always transform a given model to use variables with a different coding; but as a function of $\mathbf{y}$ it may look different than the original model in $\mathbf{z}$.

If, conversely, we just plug y into the original model (possibly with a different parameter value), we get an alternative PMF for $\mathrm{Y}$ :

$$
f_{\mathbf{Y}}^{\prime} \propto g\left(\mathbf{y} ; \boldsymbol{\theta}^{\prime}\right)=g\left(a \mathbf{z}+b \mathbf{1} ; \boldsymbol{\theta}^{\prime}\right) .
$$

In order to achieve $f_{\mathbf{Y}}=f_{\mathbf{Y}}^{\prime}$, we must have $g(\mathbf{z} ; \boldsymbol{\theta}) \propto g(a \mathbf{z}+$ $\left.b \mathbf{1} ; \boldsymbol{\theta}^{\prime}\right)$. There is no guarantee that $\boldsymbol{\theta}^{\prime}$ can be chosen to make them proportional; it depends on the algebraic form of $g$. This is exactly the rationale followed in the proofs of Theorems 1 and 2.

\section{B. THE AUTOLOGISTIC MODEL WITH ARBITRARY CODING}

The goal is to derive expressions for the joint PMF, the conditional PMFs, and the conditional $\log$ odds of variables $\mathbf{Z}$, in the situation where the coding is $\{\ell, h\}$. Caiser and Cressie [3] and Hughes et al. [25] provide formulae for constructing the joint PMF (up to a normalizing constant) given the conditionals:

$$
\begin{gathered}
f_{\mathbf{Z}}(\mathbf{z}) \propto e^{Q(\mathbf{z})} \\
Q(\mathbf{z})=\sum_{i=1}^{n} G_{i}\left(z_{i}\right)+\sum_{(i, j) \in \mathcal{E}} G_{i j}\left(z_{i}, z_{j}\right) \\
G_{i}\left(z_{i}\right)=\log \left(\frac{f_{i}\left(z_{i} \mid \mathbf{z}_{-i}^{*}\right)}{f_{i}\left(z_{i}^{*} \mid \mathbf{z}_{-i}^{*}\right)}\right)
\end{gathered}
$$

$$
G_{i j}\left(z_{i}, z_{j}\right)=\log \left(\frac{f_{i}\left(z_{i} \mid z_{j}, \mathbf{z}_{-i j}^{*}\right)}{f_{i}\left(z_{i}^{*} \mid z_{j}, \mathbf{z}_{-i j}^{*}\right)} \frac{f_{i}\left(z_{i}^{*} \mid \mathbf{z}_{-i}^{*}\right)}{f_{i}\left(z_{i} \mid \mathbf{z}_{-i}^{*}\right)}\right),
$$

where $f_{\mathrm{Z}}$ is the joint PMF and $f_{i}$ is the conditional PMF of variable $i$ given the values of all other variables. In keeping with the MRF dependence structure, $f_{i}$ will be a function of only variable $i$ 's neighbors: $f\left(z_{i} \mid \mathbf{z}_{-i}\right)=f\left(z_{i} \mid \mathbf{z}_{j \sim i}\right)$.

In these expressions, $Q(\mathbf{z})$ is the negpotential function; $\mathbf{z}_{-i}$ and $\mathbf{z}_{-i j}$ are vectors of all the variables excluding variables $i$ and $i, j$ respectively; and $\mathbf{z}^{*}$ is a chosen value of $\mathbf{z}$ in the support of $f_{\mathbf{Z}}(\mathbf{z})$. A necessary and sufficient condition on the support sets of the random variables, which ensures that the above-defined $f_{\mathbf{Z}}(\mathbf{z})$ is a valid PMF, is given in Kaiser and Cressie [3]. The condition is satisfied in our case.

Framework (A1-A4) allows the joint PMF to be built up from a specification of the conditionals. Let $\pi_{i}=f_{i}\left(h \mid \mathbf{z}_{-i}\right)=$ $\operatorname{Pr}\left(Z_{i}=h \mid \mathbf{z}_{-i}\right)$. Since we are not using the traditional $\{0,1\}$ support for $z_{i}$, we cannot write the conditional PMF as $f_{i}\left(z \mid \mathbf{z}_{-i}\right)=\pi_{i}^{z}\left(1-\pi_{i}\right)^{1-z}$. Instead, write

$$
f_{i}\left(z \mid \mathbf{z}_{-i}\right)=\frac{e^{r(z)}}{e^{r(h)}+e^{r(\ell)}},
$$

with $r(z)$ to be specified. In this way $f_{i}\left(\ell \mid \mathbf{z}_{-i}\right)=1-f_{i}\left(h \mid \mathbf{z}_{-i}\right)$ as it should, and the conditional log odds expression is

$$
\operatorname{logit}\left(\pi_{i}\right)=\log \frac{f_{i}\left(h \mid \mathbf{z}_{-i}\right)}{f_{i}\left(\ell \mid \mathbf{z}_{-i}\right)}=r(h)-r(\ell) .
$$

Now define $r\left(z_{i}\right)$ to be

$$
r\left(z_{i}\right)=z_{i}\left(\alpha_{i}+\sum_{j \sim i} \lambda_{i j}\left(z_{j}-\mu_{j}\right)\right),
$$

where $\mu_{j}$ is either the independence expectation of $z_{j}$ in the centered case, or zero in the standard case. From this we arrive at the centering adjustment (7), the conditional PMF (9), and the conditional $\log$ odds (10).

To work out the joint PMF of $\mathbf{Z}$, use the $f_{i}$ functions just defined in formulas (A3) and (A4). It is easiest to work with the joint PMF in matrix/vector form, so let $\boldsymbol{\Lambda}, \boldsymbol{\alpha}$, and $\boldsymbol{\mu}$ be as defined in section 2.3, and define $\boldsymbol{\Lambda}_{i}$ to be the ith column of $\boldsymbol{\Lambda}$. Also choose $\mathbf{z}^{*}=\ell$, a vector with all its elements equal to $\ell$. We find that

$$
G_{i}\left(z_{i}\right)=\alpha_{i}\left(z_{i}-\ell\right)+\left(z_{i}-\ell\right) \boldsymbol{\Lambda}_{i}^{T}(\boldsymbol{\ell}-\boldsymbol{\mu})
$$

and

$$
G_{i j}\left(z_{i}, z_{j}\right)=\left(z_{i}-\ell\right) \lambda_{i j}\left(z_{j}-\ell\right) .
$$

Combining these $G$ functions as in Equation (A2), we obtain

$$
Q(\mathbf{z})=(\mathbf{z}-\ell)^{T} \boldsymbol{\alpha}+(\mathbf{z}-\boldsymbol{\ell})^{T} \boldsymbol{\Lambda}(\boldsymbol{\ell}-\boldsymbol{\mu})+\frac{1}{2}(\mathbf{z}-\boldsymbol{\ell})^{T} \boldsymbol{\Lambda}(\mathbf{z}-\boldsymbol{\ell}) .
$$

After expanding the right hand side, some terms cancel and others are free of $\mathbf{z}$ and thus can be moved into the normalizing constant. Doing this yields the negpotential function used in PMF (11). 